\title{
A Gas-poor Planetesimal Capture Model for the Formation of Giant Planet Satellite Systems
}

\author{
Paul R. Estrada ${ }^{1}$ and Ignacio Mosqueira ${ }^{1,2}$ \\ ${ }^{1}$ NASA Ames Research Center, ${ }^{2}$ SETI Institute
}

\begin{abstract}
Assuming that an unknown mechanism (e.g., gas turbulence) removes most of the subnebula gas disk in a timescale shorter than that for satellite formation, we develop a model for the formation of regular (and possibly at least some of the irregular) satellites around giant planets in a gas-poor environment. In this model, which follows along the lines of the work of Safronov et al. (1986), heliocentric planetesimals collide within the planet's Hill sphere and generate a circumplanetary disk of prograde and retrograde satellitesimals extending as far out as $\sim R_{H} / 2$. At first, the net angular momentum of this proto-satellite swarm is small, and collisions among satellitesimals leads to loss of mass from the outer disk, and delivers mass to the inner disk (where regular satellites form) in a timescale $\lesssim 10^{5}$ years. This mass loss may be offset by continued collisional capture of sufficiently small $<1 \mathrm{~km}$ interlopers resulting from the disruption of planetesimals in the feeding zone of the giant planet. As the planet's feeding zone is cleared in a timescale $\lesssim 10^{5}$ years, enough angular momentum may be delivered to the proto-satellite swarm to account for the angular momentum of the regular satellites of Jupiter and Saturn. This feeding timescale is also roughly consistent with the independent constraint that the Galilean satellites formed in a timescale of $10^{5}-10^{6}$ years, which may be long enough to accomodate Callisto's partially differentiated state (Anderson et al. 1998; 2001). In turn, this formation timescale can be used to provide plausible constraints on the surface density of solids in the satellitesimal disk (excluding satellite embryos $\sim 1 \mathrm{~g} \mathrm{~cm}^{-2}$ for satellitesimals of size $\sim 1 \mathrm{~km}$ ), which yields a total disk mass smaller than the mass of the regular satellites, and means that the satellites must form in several $\sim 10$ collisional cycles. However, much more work will need to be conducted concerning the collisional evolution both of the circumplanetary satellitesimals and of the heliocentric planetesimals following giant planet formation before one can assess the significance of this agreement. Furthermore, for enough mass to be delivered to form the regular satellites in the required timescale one may need to rely on (unproven) mechanisms to replenish the feeding zone of the giant planet. We compare this model to the solids-enhanced minimum mass (SEMM) model of Mosqueira and Estrada (2003a,b), and discuss its main consequences for Cassini observations of the Saturnian satellite system.
\end{abstract}

\section{Introduction}

The similarities between planetary and satellite systems led to early suggestions that any theory for planetary formation should incorporate regular satellite formation as 
a natural byproduct (Alfven 1971; De et al. 1977). More recently the propensity has been to argue against a unifying, generally applicable formation model for planets and satellites (e.g., Stevenson et al. 1986). Yet, whether or not regular satellites and planets formed in an analogous manner, it is often remarked that the scaled bulk characteristics of angular momentum and mass are shared by the regular satellites of Jupiter, Saturn and Uranus (e.g., Pollack et al. 1991). The first to suggest that regular satellites formed out of a collisionally-captured gravitationally-bound swarm of circumplanetary satellitesimals was O. Yu Schmidt (1957). This idea was subsequently explored in a number of publications (Safronov and Ruskol (1977); Ruskol 1981, 1982; Safronov et al. 1986). However, it is fair to say that while these works provide valuable insight into the physical mechanisms possibly involved in the formation of regular satellites of giant planets out of an extended circumplanetary satellitesimal swarm, they fall short of advancing a specific model that can account for the observed properties of the satellite systems. In this contribution, we revisit this approach with the aim of providing a testable model as well as a basis for comparison with our published solids-enhanced minimum mass (SEMM) satellite formation model (Mosqueira and Estrada 2003a,b; hereafter, MEa,b).

The primary difference between the SEMM model and the present gas-poor planetesimal capture (GPPC) model is in the treatment of turbulence. It is commonplace in the planetary formation literature to assume that Keplerian disks are turbulent and to model the resulting anomalous angular momentum transport in terms of an $\alpha \sim 10^{-3}$ parameter $^{1}$ (Shakura and Sunyaev 1973) regardless of disk conditions or distance from the central object. While this assumption may or may not (given the scatter in the data and the existence of alternative explanations) be justified in the case of the solar nebula by the observation that disks around young protostars accrete at low rates $\sim 10^{-8} M_{\odot} \mathrm{yr}^{-1}$ (Hartmann et al. 1988), there is no reason to suppose that similar $\alpha$ values need also apply to circumplanetary nebulae at the time of satellite formation. Indeed, even those turbulent mechanisms (such as MHD [Balbus and Hawley 1998] and baroclinic instabilities [Li et al. 2000; Klahr and Bodenheimer 2003]) that may operate at least at some locations and times in the case of the planetary disk may fail in the dense (but not massive), cold and mostly isothermal circumplanetary disk (MEa). ${ }^{2}$

It is often suggested that the disk's radial shear may drive turbulence for sufficiently high Reynolds number (Zahn 1991; Dubrulle 1993; Richard and Zahn 1999). If true, such a mechanism would apply quite generally to accretion disks (both planetary and satellite disks would be affected); however, so far numerical simulations (Hawley et al. 1999; Mosqueira et al. 2003) have failed to show the presence of a non-linear shear instability, apparently due to the stabilizing Coriolis terms in flows with posi-

\footnotetext{
${ }^{1}$ It should be kept in mind, however, that some workers favor the $\beta$ parameter (i.e., Richard and Zahn 1999)

${ }^{2}$ One would expect subnebula turbulence while there is significant Roche-lobe gas inflow, but it is difficult to see how this source of turbulence can be relied upon to remove the circumplanetary gas disk.
} 
tive radial gradients in specific angular momentum (cf. Rayleigh stable flows), even in the presence of both a radial and a vertical shear (Rudiger et al. 2002). In fact, it has been claimed that a carefully constructed Taylor-Couette experiment shows stability in the case of positive radial gradients in specific angular momentum even for high Reynolds number (Schultz-Grunow 1959) ${ }^{3}$. The difficulty stems not only from the degree of turbulence, but also whether such turbulence, if present, may be characterized by an $\alpha$ parameter.

Given the lack of observational or theoretical quantitative constraints for the gas inflow rate through the gap or planetary accretion rate in the final stages of giant planet formation (Lubow et al. 1999), characterization of the subnebula evolution at the time of satellite formation is clearly beyond reach at the present time. One may tackle this hurdle either by assuming that turbulence decays so that Keplerian disks must pass through quiescent phases (which enables planetesimal and satellitesimal formation), or by assuming that satellites begin to form once the subnebula has been mostly depleted of gas (which facilitates their survival) presumably due to sustained turbulence of unknown origin (possibly hydrodynamic shear turbulence). In the former case, satellite survival hinges on gap-opening, and this mechanism itself sets the value for the gas surface density of the satellite disk (MEb). In the latter case, which is the focus of the present paper, the gas surface density is low but unspecified, though the presence of some gas may help to explain the observations. Here satellite formation is dealt with in a manner somewhat analogous to the consensus treatment for the terrestrial planets.

The second major difference between the two models concerns the mechanism controlling the timescale for satellite formation. MEa,b includes satellitesimal migration due to gas drag and tidal torques, and forms satellites by a combination of Safronovtype binary accretion and drift augmented accretion in an extended, two-component, largely quiescent subnebula. This leads to a model in which satellitesimals $(\sim 100$ $\mathrm{km})$ form quickly $(\sim 1000$ years $)$ but full grown satellites $(\sim 1000 \mathrm{~km})$ take significantly longer to form $\left(10^{4}-10^{6}\right.$ years depending on their location; Mosqueira et al. 2001), in a time set by the gas drag time of satellitesimals in a solids-enhanced minimum mass subnebula ${ }^{4}$ (enhanced by a factor $\sim 10$, which leads to a gas surface density $\sim 10^{4} \mathrm{~g} \mathrm{~cm}^{-2}$ ). Furthermore, this formation timescale is consistent with the Type I migration timescale of full grown satellites in such a disk provided 3-D effects are considered (which leads to a factor $\sim 10$ slower migration; e.g., Bate et al. 2003), and with the criterion for gap-opening in an inviscid disk (with aspect ratio $\sim 0.1$; Rafikov 2002).

In contrast, in this paper the formation timescale for the regular satellites is set

\footnotetext{
${ }^{3}$ Though it is unclear whether this would remain true for even higher Reynolds number. Experimentally, the onset of turbulence in pipe flows cannot be described simply by linear stability analysis.

${ }^{4}$ Gas flow through the giant planet's gap (Lubow et al. 1999) does not necessarily imply that the time for disk formation exceeds that of satellite formation. For instance, one possible scenario is for Jupiter and Saturn to clear the disk in a timescale $<10^{5}$ years in a weakly turbulent nebula (Bryden et al. 1999, 2000).
} 
by the timescale over which planetesimal fragments are collisionally captured by the circumplanetary satellitesimal swarm, which is ultimately tied to the timescale for a giant planet to clear its feeding zone (Mosqueira et al. 2000). Given that the gassurface density in this model is unspecified but is presumably quite small $(<100 \mathrm{~g}$ $\mathrm{cm}^{-2}$ ), the migration of satellites due to either gas drag or tidal torques does not pose a threat to their survival; yet, one may still use the gas disk to circularize the orbits of the regular satellites, or to sweep away collisional debris from regions of the disk that are observed to be either empty or significantly mass-depleted (such is the case with most of the Saturnian satellite system).

The third major difference we draw attention to in this introduction (see also the comparison table in Appendix $\mathrm{C}$ as well as MEa,b) concerns the interpretation of the compositional gradient of the Galilean satellites. Two main explanations have been suggested for this observation. A number of publications ascribe the high-silicate fraction of Europa (Io might have lost its volatiles due to the Laplace resonance alone) compared to Ganymede and Callisto to the subnebula temperature gradient due to the giant planet's luminosity at the time of satellite formation following envelope collapse (e.g., Pollack et al. 1976; Lunine and Stevenson 1982). On the other hand, Shoemaker (1984) argued that this compositional gradient might be due to the increase of impact velocities and impactor flux of Roche-lobe interlopers deep in the planetary potential well, leading to preferential volatile depletion in the case of Europa and Io. That is, in this view all the Galilean satellites started out ice-rich, but some lost more volatiles than others.

In the context of our decaying-turbulence SEMM model, the Galilean satellite compositional gradient is used to set a subnebula temperature at the location of (gap-opening) Ganymede of $T \sim 250 \mathrm{~K}$ (or aspect ratio of $\sim 0.1$ as stated above) controlled by the giant planet's luminosity. ${ }^{5}$ This may imply a planetary luminosity between $10^{-4}-10^{-5} L_{\odot}$ for a planetary radius of $1.5-2.0 R_{J}$ (where $R_{J}$ is Jupiter's radius) following envelope collapse (work in progress). Here we simply note that deep in the planetary potential well one might expect a stochastic compositional component due to high speed $\sim 10 \mathrm{~km} \mathrm{~s}^{-1}$ impacts with large $\sim 100 \mathrm{~km}$ Roche-lobe interlopers (but see Mosqueira and Estrada 2005 for the case of Iapetus, which may be exempt from this argument due to its distance from the planet, size and isolation). Such hyperbolic collisions might conceivably remove volatiles from the mantle of a differentiated satellite and place them on neighboring satellites. This might explain the volatile depletion in Europa relative to its outer neighbours (given its density [Jacobson et al. 2005], a similar argument may apply to Enceladus in the Saturnian system) and obviate the need for a temperature of formation constraint. In this paper we adopt this point of view.

Finally, it is useful to compare the method of delivery of solids in each model. We expect that the regular satellites form at the tail-end of planetary accretion so that, at least in the core accretion model for the formation of the giant planet, almost all

\footnotetext{
${ }^{5}$ It is important to stress that because this is a decaying turbulence model, the disk's temperature is determined by the planet's luminosity and not by viscous heating.
} 
of the solids mass in the solar nebula starts out in the form of $\sim 10 \mathrm{~km}$ planetesimals (e.g., Weidenschilling 1997). However, following giant planet formation fragmentation may cascade a significant fraction of the mass in the feeding zone of the giant planets back to smaller sizes (Stern and Weissman 2001; Charnoz and Morbidelli 2003). Planetesimals in the meter to kilometer mass range may deliver mass to the regular satellites of the giant planets either by ablating in the giant planet envelope prior to envelope collapse, or by ablating through the circumplanetary gas disk (MEa; Mosqueira and Estrada 2005). In the context of this paper, planetesimals in this size range are both easier to capture into the circumplanetary disk, and may also be used to replenish the feeding zone of the giant planet by gas drag.

In Section 1.1 we summarize the main points of the GPPC model. The creation of the circumplanetary satellitesimal disk is discussed in more detail starting in Section 2. Readers who are more interested in the results may wish to skip to Section 2.3. In Section 2.4, we present results of the angular momentum calculation. Finally, in Section 3 we present discussion of the results and our conclusions. Several appendices are provided at the end of the paper for reference.

\subsection{Model Outline}

In this section, we summarize the GPPC model. In the spirit of Safronov et al. (1986), we will label quantities that are related to the circumsolar disk with the subscript " 1 ", and those that are related to the circumplanetary disk with the subscript " 2 ". The reader is referred to Appendix A for a list of symbols.

1. The creation of a protosatellite "swarm" 6 will produce a disk of prograde and retrograde satellitesimals extending as far as circumplanetary orbits are stable $\sim R_{H} / 2$ (Safronov et al. 1986). Initially, the capture rate is dominated by inelastic collisions within the planet's Hill radius of similarly-sized heliocentric planetesimals (see Section 2.1). As the satellitesimal disk becomes more massive its volume density $\rho_{2}$ overtakes the heliocentric planetesimal density $\rho_{1}$. Once this happens, the dominant mass capture mechanism becomes collisions between planetesimals and larger satellitesimals (see Section 2.3).

2. The net specific angular momentum $\ell_{z}$ of this satellitesimal swarm is likely to be small; collisions between prograde and retrograde satellitesimals will result in a close-in, prograde satellite disk. Close to the giant planet, hypervelocity collisions with incoming planetesimals may shatter embryo-sized or smaller objects $\left(\sim 10^{2}-10^{3}\right.$ $\mathrm{km}$ ), and alter satellite compositions, which may ultimately result in a diversity of outcomes (i.e., Galilean-like vs. Titan-like satellite systems).

3. Collisions between outer disk satellitesimals can lead to fragmentation, accretion or removal from the outer disk to the inner disk, where this material will be accreted by satellite embryos. Removal of material from the outer disk is balanced by

\footnotetext{
${ }^{6}$ Although this terminology was used by Safronov to describe the circumplanetary disk, we shall adopt the less ambiguous choice of calling swarm objects satellitesimals.
} 
planetesimal fragment collisional capture (by larger circumplanetary satellitesimals). Here we rely on fragmentation to decrease the size of heliocentric planetesimals, which are easier to capture.

4. In the outer disk we expect that numerous $\sim 1 \mathrm{~km}$ objects (of which irregular satellites may be surviving members) contribute a low optical depth $\tau_{z 2} \sim 10^{-5}$, which yields a surface density $\sigma_{2} \sim 1 \mathrm{~g} \mathrm{~cm}^{-2}$ and volume density $\rho_{2} \sim 1.4 \times 10^{-14}\left(\sigma_{1} / \sigma_{\mathrm{MM}}\right)$ $\mathrm{g} \mathrm{cm}^{-3}$, where $\sigma_{1}=\sigma_{\mathrm{MM}}=3.3 \mathrm{~g} \mathrm{~cm}^{-2}$ corresponds to the solids surface density of a minimum mass solar nebula (MMSN; Hayashi 1981). This means that even for the case of a solids MMSN, $\sigma_{1}>\sigma_{2}$ but typically $\rho_{1}<\rho_{2}$, as noted in 1 . To avoid fast satellite formation the mass in the extended disk of solids at any one time (excluding satellite embryos) must be a small fraction of a Galilean satellite $\left(\sim 10^{26} \mathrm{~g}\right)$. This can lead to a long $10^{5}-10^{6}$ years satellite formation time (presumably consistent with Callisto's partially differentiated state) controlled by planetesimal feeding.

5. As the giant planet clears its feeding zone in a $\sim 10^{5}$ year timescale small planetesimals $^{7}$ are fed from the outer regions of the feeding zone and deliver enough angular momentum to the disk to form the regular satellites at their observed locations.

Satellitesimal disk collapse time: Given typical satellitesimals of size $\sim 1 \mathrm{~km}$, and a total disk mass a fraction $\sim 0.1$ of a Galilean satellite (which corresponds to $\sigma_{2} \sim 1$ $\mathrm{g} \mathrm{cm}^{-2}$ ), the collapse time of the disk is of order $\tau_{\text {coll }} \sim P_{2} / \tau_{z 2} \sim 10^{5}$ years, where $P_{2}$ is a characteristic orbital period of satellitesimals, and $\tau_{z 2} \sim 10^{-5}$ is the optical depth contributed by the larger particles (see Section 2.3). The outer disk provides materials to the inner disk out of which the regular satellites form. Disk material must then be replenished to the outer disk by planetesimal capture, and one might need $N_{c}=\tau_{a c c} / \tau_{\text {coll }} \sim 10$ such collisional cycles to complete the accretion of the Galilean satellites in $\tau_{a c c} \sim 10^{5}-10^{6}$ years, which may be long enough to explain Callisto's partially differentiated state.

Planetesimal capture: In order to show that a GPPC model is viable one needs to address whether such a disk would be able to capture a sufficient amount of mass to form the Galilean satellites. Safronov et al. (1986) considered the following delivery mechanisms:

- I. Collisions between planetesimals within $R_{H}$, which may have led to the formation of a circumplanetary disk.

- II. Collisions between large satellitesimals and smaller planetesimals, which may result in capture of the planetesimal and a mass inflow rate, $I_{l b}$, and collisions between planetesimals and many smaller satellitesimals, which may result in capture of the planetesimal (if it encounters a mass comparable to itself) and a mass inflow rate $I_{s p}$.

\footnotetext{
${ }^{7}$ This population of objects deserve special treatment since they will have smaller inclinations than eccentricities. Ohtsuki and Ida (1998) discuss the effects of small planetesimals on planetary spin rates.
} 
- III. Erosion or delivery of ejecta from a collision of a planetesimal with a smaller satellitesimals, which may provide a mass inflow rate, $I_{e}$.

The first method of mass delivery (I) is addressed in the formation of the circumplanetary disk in Section 2.1. As indicated, this mechanism is dominant until the solids volume density in the satellitesimal disk $\rho_{2}$ exceeds that of the solar nebula, $\rho_{1}$. In our model mechanism (II) determines whether enough mass can be delivered to the system in the planetesimal feeding time. Although mechanism (III) may provide a source of material, the amount of mass captured by collisional erosion is uncertain ${ }^{8}$, so we do not use $I_{e}$ or address it any further. We can estimate the mass delivered to the disk on a timescale of $\tau_{a c c} \sim 10^{6}$ years using (see Section 2.3)

$$
M=2 \pi \tau_{a c c} \int_{R_{0}}^{R_{D}} I(R) p(R) R d R,
$$

where $I(R)$ is the total mass inflow collision rate per unit area, $p(R)$ is a capture probability, and $R_{0}$ and $R_{D}$ define the inner and outer edges of the circumplanetary disk, respectively. Unless otherwise noted, we generally take $R_{0}$ to be the radius of the planet $R_{P}$, and $R_{D}=R_{H} / 2$. The inflow rate $I(R)$ can be expressed in terms of the disk optical depth $\tau_{z 2}$, solar nebula solids density $\rho_{1}$, impact velocity of incoming planetesimals $v_{1}$, and the planetesimal and satellitesimal cutoff radii $r_{1, \max }, r_{2, \max }$ (Safronov et al. 1986), where (see Section 2.3)

$$
I(R)=I_{s p}+I_{l b}=\rho_{1} v_{1} \tau_{z 2}\left(\frac{r_{2, \max }}{r_{1, \max }}\right)^{1 / 2}\left[\tau_{z 2}-\ln \tau_{z 2}\right]
$$

and a mass distribution with power law exponent of $q_{1}=q_{2}=11 / 6$ has been assumed for both the planetesimal and satellitesimal populations. Here, $\tau_{z 2}=3 \sigma_{2} / 4 \rho_{s} r_{2, \text { max }}=$ $\pi r_{2, \max }^{2} \sigma_{2} / m_{2, \max }$ is the vertical optical depth ${ }^{9}$ of the satellitesimal disk $\left(\rho_{s}\right.$ is the satellitesimal density), which is assumed to be set by the size of the largest satellitesimal $r_{2, \max }$ (Safronov et al. 1986). Ruskol (1975) gives a functional form for the probability distribution $p(R)$ which measures the probability that a collision will result in capture (as supposed to fragmenting and scattering into escape orbits; see Section 2.1)

$$
p(R)=\frac{\theta_{1}}{l+2 \theta_{1}}\left(1-R / R_{H}\right),
$$

where $\theta_{1}$ is the planetesimals Safronov parameter, and $l=R / R_{P}$. We can then evaluate the mass delivered by planetesimals primarily striking larger or comparablesized satellitesimals and obtain

\footnotetext{
${ }^{8}$ Note that capture of material due to erosion differs from larger planetesimals captured into the circumplanetary disk by smaller satellitesimals, which we treat in Section 2.3.

${ }^{9}$ This definition of the optical depth is only true if all the particles in the disk are of size $r_{2, \max }$. For this reason, $\tau_{z 2}$ should be considered more a probability of collision with a satellitesimal of size $r_{2, \max }$ than an optical depth.
} 


$$
M \sim 10^{26}\left(\frac{\sigma_{1}}{3.3 \mathrm{~g} \mathrm{~cm}^{-2}} \frac{\sigma_{2}}{1 \mathrm{~g} \mathrm{~cm}^{-2}}\right)\left(\frac{10 \mathrm{~km}}{r_{1, \max }} \frac{1 \mathrm{~km}}{r_{2, \max }}\right)^{1 / 2} \mathrm{~g} .
$$

This is comparable to a Galilean satellite mass for reasonable choices of parameters (but see Section 2.3 for further discussion).

However, an outstanding issue is whether planetesimals may be fed into the system over a timescale $\gtrsim 10^{5}$ years. This is because the giant planet may clear planetesimals from its feeding zone on a shorter timescale. The formation of this gap was not taken into account in the above estimate of the planetesimal capture rate. This can be seen in the following simple way. A reasonable estimate for the total amount of mass in planetesimals in the feeding zone of the giant planets is $\sim 10 M_{\oplus} \sim 10^{29} \mathrm{~g}$. Suppose we assume that every planetesimal and satellitesimal has a size of $1 \mathrm{~km}$, and that inelastic collisions between them always lead to planetesimal capture into circumplanetary orbit. Then we can ask how many times $n_{p}$ will a planetesimal cross the circumplanetary disk within $R_{H} / 2$ before being scattered by the giant planet. Ignoring gravitational focusing and taking the feeding zone of the giant planet to be of width $4 \sqrt{3} R_{H}$, we obtain

$$
n_{p}=\frac{0.1}{576 \sqrt{3} \pi}\left(\frac{a}{R_{H}}\right)^{5} \sim 10-100 .
$$

Given an optical depth of $\tau_{z 2} \sim 10^{-5}$ the total amount of material captured is $\sim 10^{26}$ g. But this material would be fed in a timescale

$$
\tau_{e j} \sim \frac{0.1}{\Omega_{1}}\left(\frac{M_{\odot}}{M_{J}}\right)^{2} \sim 10^{5} \text { years }
$$

which may be too short given our chosen system constraints. Also, since it is unrealistic to assume perfect capture $(i . e ., p(R)<1)$ and accretion efficiencies, one may have to replenish the feeding zone of the giant planet with planetesimal fragments brought in by gas drag and enhance the solid content of the nebula with respect to the minimum mass solar nebula. Although we discuss this issue in Section 3, further work will need to be done to assess whether this is feasible.

Angular momentum of the satellitesimal disk: A key issue is whether the delivery of planetesimals provides sufficient angular momentum to the disk to account for the angular momentum budget of the regular satellites of the giant planets. Assuming that a significant fraction of the mass is in small planetesimals $r_{1}<1 \mathrm{~km}$ with large Safronov parameter $\theta_{1}>1$ (so that $p(R) \lesssim 1$ ), we can calculate the amount of angular momentum delivered to the satellitesimal disk as the giant planet clears its feeding zone. A rough estimate of the angular momentum budget may be obtained assuming a Rayleigh distribution of planetesimal velocities and neglecting the gravitational effects of the planet. The specific angular momentum delivered by planetesimals is given by (see Section 2.4) 


$$
\left\langle\ell_{z}\right\rangle=\frac{2 \pi}{\dot{M}} \int_{R_{0}}^{R_{D}} \epsilon \mathcal{I}_{L}(R) R d R
$$

and the mass inflow rate is

$$
\dot{M}=2 \pi \int_{R_{0}}^{R_{D}} \mathcal{I}(R) p(R) R d R
$$

where $\mathcal{I}_{\mathcal{L}}(\mathcal{R})$ is the angular momentum delivery rate per unit area given perfect angular momentum deposition efficiency $\epsilon=1$, and $\mathcal{I}(R)$ is the mass collision inflow rate per unit area. This gives a net specific angular momentum that is too small to create a disk of sufficient size to make the satellites unless most of the planetesimals originate from the outer regions of the planet's feeding zone. If we use a distribution of semi-major axes depleted inside an annulus of half-width $\sim 1 R_{H}$ centered on the giant planet, and a velocity dispersion of planetesimals $v_{1} \sim 6 \mathrm{~km} \mathrm{~s}^{-1}\left(\theta_{1} \sim 100\right)$, we find (see Section 2.4)

$$
\left\langle\ell_{z}\right\rangle \sim 0.3 \Omega R_{H}^{2} \rightarrow R_{\text {coll }}=\frac{\left\langle\ell_{z}\right\rangle^{2}}{G M_{J}} \sim 20 R_{J},
$$

where $R_{\text {coll }}$ is the centrifugal collapse radius, and $M_{J}$ is the mass of Jupiter. This suggests that it may be possible for the extended disk of prograde and retrograde satellitesimals to collapse to the observed Galilean satellite system size.

\section{Planetesimal Model}

Turbulence may act both to remove gas from the subnebula and to replenish it by feeding gas through the giant planet's gap. However, we do not rely on this gas inflow as a source of material to make satellites for several reasons. First, the inflow rate is nearly unconstrained beyond the fact that it must be very weak if ice-rich satellites are to survive (orders of magnitude slower than in Lubow et al. 1999; see, e.g., Makalkin et al. 1999, Canup and Ward 2002, Alibert and Mousis 2004). Second, the high-specific angular momentum of this component would lead to the formation of an extended disk around the planet ${ }^{10}$, whereas regular satellites are observed to be close-in. Third, the solid content of this gas component is unknown, and a significant fraction of this material might accrete onto the planet along with the gas. Fourth, most of the mass of solids in the planetary disk is likely to be in objects larger than the gas coupling size, which means that planetesimal dynamics should be treated.

\footnotetext{
${ }^{10} \mathrm{It}$ is important to note that slower inflow rates correspond to larger gaps and higher specific angular momentum for the inflow.
} 


\subsection{Formation of the Circumplanetary Satellitesimal Swarm}

Planetesimal circumplanetary collisional (or collisionless) capture can be investigated as a three-body problem with planetesimals treated as massless particles. Inelastic collisions of heliocentric planetesimals within the Hill sphere of the giant planet can lead to capture of solids because inelastic collisions decrease relative velocities. In the 3-body problem one can define the origin to lie at the center of mass of the system, and write down the Jacobi constant as

$$
C_{J}=\Omega_{1}^{2}\left(x^{2}+y^{2}\right)+\frac{2 G M_{\odot}}{a}+\frac{2 G M_{P}}{R}-v^{2},
$$

Inelastic collisions can increase this quantity (by decreasing $v^{2}$ ) to values indicative of a circumplanetary orbit, which allows for the possibility of planetesimal capture by the planet's gravitational field. Here the $x$-axis is directed along the line connecting the finite masses, $a$ is the planetesimals distance from the Sun, $R$ its distance from the giant planet, and $\Omega_{1}$ is the uniform angular velocity of the system. Planetesimal collisions are inelastic because there is loss of kinetic energy through a number of mechanisms such as fragmentation, heating, melting, evaporation, and the like. Thus, collisions can occur that lead to a change in velocity $0<|\Delta v|<v$ such that the variation in the Jacobi constant (constant absent a collision) is $\Delta C_{J} \sim-2 v \Delta v \gtrsim 0$.

We can obtain an analytical estimate of this change in energy by assuming that similar-sized particles collide while travelling at similar speeds $v \sim v_{10}=\sqrt{G M_{P} / \theta_{1} R_{P}}$. Scaling the system to units such that the gravitational constant $G=1$, distances are in $\mathrm{AU}$, masses are in terms of the mass ratio of secondary to primary $\mu \equiv M_{P} / M_{\odot}$, and time in terms of the orbital period ( $1 \mathrm{AU}$ ) over $2 \pi$, then in the case of Jupiter one finds

$$
\Delta C_{J} \approx v^{2} \approx 2 \times 10^{3} \frac{\mu}{\theta_{1}} \sim 0.7-0.4 \text { for } \theta_{1}=3-5
$$

We can compare this to the difference in the Jacobi constant of circular orbits at $30 R_{J}$ (a representative size of the Jovian satellite system) with respect to the L1 Lagrange point $\Delta C_{J} \sim 0.04$. Thus, inelastic collisions likely allow for the capture of planetesimals for Jacobi constants close to the value at L1 into orbits like the Galilean satellites with capture into orbits at further distances even more energetically feasible.

However, mass differences between the colliding particles, as well as their initial energies leads us to introduce a capture probability. Ruskol (1975) derives a heuristic capture probability that applies to collisions between similar-sized particles, which she defines as those particles whose masses do not differ by more than a factor of two. Incoming planetesimals are assumed to have speed $v_{10}$ at infinity and as they approach the giant planet they reach a speed $v_{1}=\sqrt{v_{10}^{2}+v_{e}^{2}}$, where $v_{e}=\sqrt{2 G M_{P} / R}$. The velocity vectors at infinity and at distance $R$ are randomly oriented. For a collision taking place at $R$, loss of energy from the collision allows for capture of the colliding bodies or their collisional fragments into circumplanetary orbits if they retain a kinetic 
energy fraction $f_{C}<\left(v_{e} / v_{1}\right)^{2}$, or more simply if their speed after the collision is less than the escape speed at distance $R$. The condition for capture is (Ruskol 1975)

$$
f_{C}<\frac{2 \theta_{1} R_{P}}{R+2 \theta_{1} R_{P}}
$$

This indicates that the allowable fraction of post-collisional kinetic energy decreases with distance from the planet. Assuming that the probability of capture is proportional to this term, we can write

$$
p(R)=\beta f_{C}\left(1-R / R_{H}\right)
$$

where the parameter $\beta<1$ accounts for the average fractional amount of material captured in the collision. For our model, we assume $\beta=1 / 2$; however, this parameter remains uncertain and depends on physical processes which we do not address here. According to this heuristic expression, the probability of capture is highest close to the planet and vanishes at the planet's Hill radius ${ }^{11}$.

We can now estimate the amount of mass that can be delivered to the circumplanetary disk via "free-free" collisions (i.e., collisions between heliocentric planetesimals occuring within the Hill radius of the planet, as supposed to "free-bound" collisions between heliocentric planetesimals and circumplanetary satellitesimals). As pointed out by Ruskol (1975), inelastic collisions between heliocentric planetesimals of disparate masses will not contribute significantly to the delivery of mass to the circumplanetary disk. Hence, one need only consider collisions between planetesimals of comparable mass in the range $m / \chi<m<\chi m$, where $\chi \sim 2$. For the planetesimal mass spectrum, we use a power law of the form $n(m)=C m^{-q}$, where the constant $C$ can be determined by the condition for the volume density of planetesimals:

$$
\rho=\int n(m) m d m
$$

For the case of the circumsolar disk we have

$$
C_{1}=\rho_{1} \times\left\{\begin{array}{ll}
\frac{2-q_{1}}{m_{1, \max }^{2-q_{1}}-m_{1, \min }^{2-q_{1}}} & \text { for } q_{1} \neq 2 ; \\
{\left[\ln \left(m_{1, \max } / m_{1, \min }\right)\right]^{-1}} & \text { for } q_{1}=2 .
\end{array},\right.
$$

where $m_{1, \min }$ and $m_{1, \max }$ are the lower and upper cutoffs of the planetesimal mass distribution. An analogous expression can be derived for the satellitesimal distribution. One might expect that at the time of giant planet formation, most of the mass of solids will be in planetesimals in the $1-10 \mathrm{~km}$ size range (Weidenschilling 1997). This may change significantly as the solids in the solar nebula evolve due to

\footnotetext{
${ }^{11}$ We note that it may be energetically possible for collisions between comparable-sized planetesimals just outside the Hill sphere to be captured. However, in this paper, we restrict ourselves to the condition that only collisions within the Hill sphere can lead to capture as explicitely assumed in the heuristic expression in Eq. (13).
} 
collisional grinding following giant planet formation, but we still use this size range as a reference (see Section 2.3 for more discussion). At any rate, it is fair to say that smaller planetesimals make our model more probable.

As the lower limit, we take $m_{1, \min }$ to be the gas decoupling size at the location of the giant planet. This is the size for which $\Omega_{1} t_{s} \sim 1$, where $t_{s}$ is the stopping time. The drag force on a particle depends on its size $r$ and the drag regime. The mean free path of a gas molecule is $\lambda_{G} \approx(a / 1 \mathrm{AU})^{11 / 4} \mathrm{~cm}$ for the standard minimum mass solar nebula model (e.g., Hayashi 1981), which gives $\lambda_{G} \sim 1 \mathrm{~m}$ at the location of Jupiter. The stopping time is $t_{s}=\rho_{s} r / \rho c$ (Weidenschilling 1977), where $\rho$ and $c$ are the local gas density and sound speed. At the location of Jupiter, the decoupling size is $\sim 1$ $m$ in the Epstein flow regime.

The probability of a planetesimal collision per unit length is given by

$$
\mathcal{Y}(m)=\int_{m / \chi}^{\chi m} \xi \pi r^{\prime 2} n_{1}\left(m^{\prime}\right) d m^{\prime}=\left(\frac{3}{4 \pi \rho_{s}}\right)^{2 / 3} \frac{\xi \pi \rho_{1}\left(2-q_{1}\right)}{q_{1}-5 / 3} \gamma_{1} \frac{m^{5 / 3-q_{1}}}{m_{1, \text { max }}^{2-q_{1}}-m_{1, \min }^{2-q_{1}}}
$$

which is valid for $q_{1}>5 / 3$. In this expression, the cross-section factor $\xi \approx 2$ for comparable-sized bodies, and $\gamma_{1}=\chi^{q_{1}-5 / 3}-\chi^{5 / 3-q_{1}}$. Given Eq. (15), the rate of planetesimal mass undergoing collisions per unit volume can be expressed as (Ruskol 1975)

$$
J_{1}=\int_{m_{1, \min }}^{m_{1, \max }} \mathcal{Y}(m) v_{1} n_{1}(m) m d m=\frac{3}{4} \frac{\rho_{1}^{2} v_{1}}{\rho_{s} r_{1, \text { eff }}} \gamma_{1} .
$$

The effective radius $r_{1, e f f}$ is given by

$$
r_{1, e f f}= \begin{cases}\frac{\left(q_{1}-5 / 3\right)\left(11-6 q_{1}\right)}{3 \xi\left(2-q_{1}\right)^{2}} \frac{\left(r_{1, \max }^{6-3 q_{1}}-r_{1, \min }^{6-3 q_{1}}\right)^{2}}{r_{1, \max }^{11-6 q_{1}}-r_{1, \min }^{11-6 q_{1}}} & q_{1}<11 / 6 ; \\ \frac{2}{\xi} \frac{\left(r_{1, \max }^{1 / 2}-r_{1, \min }^{1 / 2}\right)^{2}}{\ln \left(r_{1, \max } / r_{1, \min }\right)} & q_{1}=11 / 6 ; \\ \frac{\left(q_{1}-5 / 3\right)\left(6 q_{1}-11\right)}{3 \xi\left(2-q_{1}\right)^{2}} \frac{\left(r_{1, \max } r_{1, \min }\right)^{6 q-11}\left(r_{1, \max }^{6-3 q_{1}}-r_{1, \min }^{6-3 q_{1}}\right)^{2}}{r_{1, \max }^{6 q_{1}-11}-r_{1, \min }^{6 q_{1}-11}} & q_{1}>11 / 6 .\end{cases}
$$

Depending on the value of the power law exponent $q_{1}, r_{1, \text { eff }}$ can be determined by the maximum particle size $\left(q_{1} \leq 11 / 6\right)$, or the minimum particle size $\left(q_{1}>11 / 6\right)$. In the case $q_{1}=11 / 6$, most of the mass (a fraction $f_{m}=1-\left(r / r_{1, \max }\right)^{3\left(2-q_{1}\right)} \sim 70 \%$ ) lies in the top decade of the mass distribution (e.g., $\sim 1-10 \mathrm{~km}$ for $r_{1, \max }=10 \mathrm{~km}$ ).

The flux of material $\dot{\mathcal{F}}_{1}$ captured into the circumplanetary disk per unit volume per unit time due to free-free collisions is then the product of Eq. (13) and Eq. (17). For $q_{1}=11 / 6$, the mass delivery rate per unit volume can then be written as

$$
\dot{\mathcal{F}}_{1}=p_{1}(R) J_{1}=p_{1} \rho_{1} v_{1} \gamma_{1} \frac{\tau_{z 1}}{H_{1}} \ln \left(\frac{r_{1, \max }}{r_{1, \min }}\right)
$$


which has been written in terms of the local vertical optical depth of solids in the nebula $\tau_{z 1}=3 \sigma_{1} / 4 \rho_{s} r_{1}$ (see footnote 9 ), where $\sigma_{1}=\rho_{1} H_{1}$ is the surface density of solids in the planetary zone, $H_{1}=\sqrt{2} v_{1} / \Omega \approx P_{1} v_{1} / 4$ is the local scale-height of solids in the nebula, and $P_{1}$ is the planet's orbital period. A rough estimate of the amount of mass delivered per year to the circumplanetary disk is

$$
\begin{array}{r}
\dot{M}_{1}=\int \dot{\mathcal{F}}_{1} d V \approx 24 \pi \gamma_{1} \frac{\left(\sigma_{1} \theta_{1} R_{J}\right)^{2}}{P_{1} \rho_{s} r_{1, \text { max }}} \ln \left(\frac{r_{1, \max }}{r_{1, \text { min }}}\right) \int_{1 / 2 \theta_{1}}^{x_{H}} \frac{x}{1+x}\left(1-x / x_{H}\right) d x \\
\sim 2 \times 10^{18}\left(\frac{\sigma_{1}}{3.3 \mathrm{~g} \mathrm{~cm}^{-2}}\right)^{2}\left(\frac{10 \mathrm{~km}}{r_{1, \text { max }}}\right) \mathrm{g} \mathrm{yr}^{-1}
\end{array}
$$

where $x_{H}=R_{H} / 2 \theta_{1} R_{P}, \rho_{s}=1 \mathrm{~g} \mathrm{~cm}^{-3}$, and we assume $\theta_{1}=4$. The timescale for the giant planet to clear its feeding zone is $\Delta t \sim 10^{5}$ years (e.g., Tanaka and Ida 1997; Charnoz and Morbidelli 2003), so this is presumably the timescale in which the circumplanetary disk should be populated. Assuming our fiducial choices for the disk parameters, one finds that the total mass delivered then is $\Delta t \times \dot{M}_{1} \sim 10^{23} \mathrm{~g}$, which is considerably smaller than a Galilean satellite. This would seem to pose a problem; however, we show below that once the circumplanetary disk becomes sufficiently massive, capture of planetesimals by inelastic collisions with circumplanetary disk particles (free-bound collisions) becomes the dominant mass delivery mechanism.

\subsection{Planetesimal Capture Due to the Circumplanetary Disk}

The capture of material due to free-free collisions may form a circumplanetary disk around the giant planet. As the density of the circumplanetary disk $\rho_{2}$ increases, it may eventually become larger than $\rho_{1}$. Once this happens, the primary delivery mechanism may become free-bound collisions (Ruskol 1975, Safronov et al. 1986).

Along the lines of the prior section, except that satellitesimals are confined to a circumplanetary disk with scale-height $\mathrm{H}_{2}$, we first estimate the mass rate per unit area undergoing collisions between planetesimals and satellitesimals of comparable size. In analogy to Eq. (16), the probability of a collision of a planetesimal crossing the circumplanetary disk in the normal direction is given by

$$
\mathcal{E}(m)=\int_{m / \chi}^{\chi m} \xi \pi r^{\prime 2} H_{2} n_{2}\left(m^{\prime}\right) d m^{\prime}=\left(\frac{3}{4 \pi \rho_{s}}\right)^{2 / 3} \frac{\xi \pi \sigma_{2}\left(2-q_{2}\right)}{q_{2}-5 / 3} \gamma_{2} \frac{m^{5 / 3-q_{2}}}{m_{2, \text { max }}^{2-q_{2}}-m_{2, \text { min }}^{2-q_{2}}}
$$

which is valid for $q_{2}>5 / 3$. Here, $n_{2}=C_{2} m^{-q_{2}}$ is the mass distribution of satellitesimals with the coefficient $C_{2}$ defined as in Eq. (15), $\gamma_{2}=\chi^{q_{2}-5 / 3}-\chi^{5 / 3-q_{2}}$, and $H_{2}$ and $\sigma_{2}$ are the scale-height and surface density of solids in the protosatellite disk, respectively. The total number of planetesimals impacting the circumplanetary disk per unit area over the mass interval $m$ to $m+d m$ is $v_{1} n_{1}(m) d m$. Integration over $m$ gives 
the mass rate per unit area of collisions between planetesimals and satellitesimals of comparable size

$$
I_{2}=\int_{m_{2, \min }}^{m_{2, \max }} \mathcal{E}(m) v_{1} n_{1}(m) m d m=\frac{3}{4} \frac{\rho_{1} \sigma_{2} v_{1}}{\rho_{s} r_{2, e f f}} \gamma_{2} \approx H_{2} J_{2},
$$

The effective radius $r_{2, e f f}$ can be expressed in terms of the appropriate limits

$$
\begin{aligned}
& r_{2, e f f}=\mathcal{R} \begin{cases}\frac{\left(11 / 3-q_{1}-q_{2}\right)\left(q_{2}-5 / 3\right)}{\xi\left(2-q_{1}\right)\left(2-q_{2}\right)} \frac{1}{r_{2, \max }^{\left(11-3 q_{1}-3 q_{2}\right)}-r_{2, \min }^{\left(11-3 q_{1}-3 q_{2}\right)}} & q_{1}+q_{2}<11 / 3 ; \\
\frac{q_{2}-5 / 3}{3 \xi\left(2-q_{1}\right)\left(2-q_{2}\right)} \frac{1}{\ln \left(r_{2, \max } / r_{2, \min }\right)} & q_{1}+q_{2}=11 / 3 ; \\
\frac{\left(q_{1}+q_{2}-11 / 3\right)\left(q_{2}-5 / 3\right)}{\xi\left(2-q_{1}\right)\left(2-q_{2}\right)} \frac{\left(r_{2, \max } r_{2, \min }\right)^{11-3 q_{1}-3 q_{2}}}{r_{2, \max }^{\left(11-3 q_{1}-3 q_{2}\right)}-r_{2, \min }^{\left(11-3 q_{1}-3 q_{2}\right)}} & q_{1}+q_{2}>11 / 3 ;\end{cases} \\
& \mathcal{R} \equiv\left(r_{1, \text { max }}^{6-3 q_{1}}-r_{1, \text { min }}^{6-3 q_{1}}\right)\left(r_{2, \text { max }}^{6-3 q_{2}}-r_{2, \text { min }}^{6-3 q_{2}}\right) .
\end{aligned}
$$

The choice of $r_{2, \min }$ is problematic given that the gas surface density in the circumplanetary disk is left unspecified. We shall, unless otherwise noted, arbitrarily choose this size to be $\sim 1 \mathrm{~cm}$, though our results are not dependent on this parameter given our choices for $q_{2}=11 / 6$ and $r_{2, \max } \sim 1 \mathrm{~km}$.

We now consider the conditions under which capture by the circumplanetary disk begins to dominate the capture rate. We can express the capture rate of material due to collisions of comparable-sized bodies as

$$
\dot{\mathcal{F}}_{c s}=\dot{\mathcal{F}}_{1}+\dot{\mathcal{F}}_{2}=p_{1} J_{1}+\frac{p_{1}}{2} J_{2}=\frac{3}{4} \frac{\rho_{1} v_{1}}{\rho_{s}}\left(\frac{\rho_{1}}{r_{1, \text { eff }}} \gamma_{1}+\frac{\rho_{2}}{2 r_{2, \text { eff }}} \gamma_{2}\right),
$$

where we have assumed that the typical amount of mass involved in a free-bound collision is roughly half that of the free-free case (Ruskol 1975). This is because we are only considering comparable-sized collisions for both capture rates. From Eq. (25), we see that in the case of collisions between particles of comparable size the condition that the dominant capture mechanism involves the circumplanetary disk is

$$
\rho_{2} \gtrsim 2 \rho_{1} \frac{\gamma_{1}}{\gamma_{2}} \frac{r_{2, \text { eff }}}{r_{1, \text { eff }}}=2 \rho_{1}\left(\frac{r_{2, \max }}{r_{1, \max }}\right)^{1 / 2} \frac{\ln \left(r_{1, \max } / r_{1, \min }\right)}{\ln \left(r_{2, \max } / r_{2, \text { min }}\right)} \sim \rho_{1},
$$

where the next to last term on the right side applies for $q_{1}=q_{2}=11 / 6$. Independent of the uncertainty in the lower bound of the size distribution for satellitesimals, we see that the circumplanetary disk dominates the capture of planetesimals when the volume density of solids in the disk becomes comparable to that of the solar nebula (Safronov et al. 1986).

To determine the implied volume density of solids in the circumplanetary disk that satisfies this condition, we estimate the mean surface density of solids in the protosatellite disk to be 


$$
\sigma_{2}=\frac{4 M_{d}}{\pi\left(R_{H}^{2}-4 R_{P}^{2}\right)} \sim \frac{4 \dot{M}_{1} \Delta t}{\pi\left(R_{H}^{2}-4 R_{P}^{2}\right)} \sim 0.01 \mathrm{~g} \mathrm{~cm}^{-2}
$$

where we have taken the mass delivered in $\Delta t=10^{5}$ years due to both mechanisms to be approximately $\dot{M}_{1} \Delta t$, we have made use of Eq. (20) with $\theta_{1}=4$, and we have ignored collisional removal. This means that $\sigma_{2}<<\sigma_{1}$. Since the critical density such that capture by the circumplanetary disk becomes dominant is $\rho_{2, \text { crit }}=\sigma_{2} / H_{2, \text { crit }}=$ $\rho_{1}$, for our disk parameters the scale-height of solids in the circumplanetary disk must be less than

$$
H_{2, \text { crit }} \lesssim 100\left(\frac{\sigma_{1}}{3.3 \mathrm{~g} \mathrm{~cm}^{-2}}\right)\left(\frac{10 \mathrm{~km}}{r_{1, \max }}\right) R_{J}
$$

and the critical density must be greater than

$$
\rho_{2, \text { crit }} \gtrsim 1.4 \times 10^{-14}\left(\frac{\sigma_{1}}{3.3 \mathrm{~g} \mathrm{~cm}^{-2}}\right) \mathrm{g} \mathrm{cm}^{-3}
$$

We stress that this is the condition for disk capture to become dominant. Once this happens the subnebula surface and volume densities may increase significantly. As we show later, in general $\sigma_{2} \lesssim \sigma_{1}$ to be consistent with chosen system constraints.

\subsection{Total Mass Delivered to the Circumplanetary Disk}

We now remove the requirement that colliding particles be of comparable mass, calculate the mass inflow rate due to the entire mass spectrum, and then use this quantity to estimate the mass delivered to the circumplanetary disk over the planetesimal feeding timescale.

Here there are two cases to consider. For a given planetesimal of mass $m$, we estimate the mass inflow collision rates per unit area (henceforth inflow rates) $I_{s p}$ and $I_{l b}$ due to interactions with satellitesimals with $m^{\prime}<m$, and $m^{\prime}>m$, respectively. These rates include the contribution due to similar-sized bodies ${ }^{12}$. In the regime we are in, we will see that the inflow rate $I_{l b}>>I_{s p}$ since the optical depth $\tau_{z 2}<<1$ given the constraints of the problem.

\footnotetext{
${ }^{12}$ In prior sections, we estimated the mass inflow rates for two mechanisms for comparable sized bodies. In the case of collisions between planetesimals in the Hill radius of the giant planet, collisions between comparable sized objects are considered because these incur the largest velocity changes resulting in an increased likelihood of capture. In the case of collisions between planetesimals and comparable-sized satellitesimals, the mass inflow rate for $q_{1}=q_{2}=11 / 6$ using Eq. (22)-(24) is

$$
I_{c s} \simeq 0.2 \frac{\rho_{1} v_{1} \sigma_{2}}{\rho_{s}\left(r_{1, \max } r_{2, \max }\right)^{1 / 2}} \ln \left(\frac{r_{2, \max }}{r_{2, \min }}\right) \simeq 0.25 \rho_{1} v_{1} \tau_{z 2}\left(\frac{r_{2, \max }}{r_{1, \max }}\right)^{1 / 2} \ln \left(\frac{r_{2, \max }}{r_{2, \min }}\right),
$$
}

which is in agreement with Eq. (6) in Safronov et al. (1986). 
For an incoming planetesimal of mass $m$ to stand a chance $p(R)$ of being captured by satellitesimals smaller than $m$, the condition is that $\pi r^{2} H_{2} \rho_{2} \geq m$ (Safronov et al. 1986), which simply says that in order for the mass $m$ to have a chance of being captured into the circumplanetary disk by particles smaller than itself, it must encounter a mass comparable or larger than itself when crossing the disk. The characteristic size for which all particles with $r<r_{c}$ stand a chance of being captured into the satellitesimal disk is

$$
r_{c} / r_{2, \max }=\left[\tau_{z 2}\left\{1-\left(r_{2, \min } / r_{c}\right)^{6-3 q_{2}}\right\}\right]^{\frac{1}{3 q_{2}-5}},
$$

where we have taken (cf. Eq. [15]) $\rho_{2}=C_{2}\left(m^{2-q_{2}}-m_{2, \text { min }}^{2-q_{2}}\right) /\left(2-q_{2}\right)$. If we assume for simplicity that $r_{c}>>r_{2, \text { min }}$, then for our fiducial case of $q_{2}=11 / 6$ one has $r_{c} \approx \tau_{z 2}^{2} r_{2, \max }$, which suggests that unless the disk solids surface density $\sigma_{2}$ is very large, only the smallest particles are assured of capture. For a given $q_{2}$ in which most of the mass is in the largest particles, the inflow collision rate is small (i.e., for $\left.\tau_{z 2}<<1\right)$. Under these circumstances, there is a probability that all bodies below this size are captured into the disk (so that $\mathcal{E}(m)=1$ ). The inflow rate on the circumplanetary disk is then just given by an integral over the number of impacting bodies on the disk's surface

$$
I_{s p}=\int_{0}^{m_{c}} v_{1} n_{1}(m) m d m \simeq \rho_{1} v_{1} \tau_{z 2}^{\frac{6-3 q_{1}}{3 q_{2}-5}}\left(\frac{r_{2, \max }}{r_{1, \max }}\right)^{6-3 q_{1}}=\rho_{1} v_{1} \tau_{z 2}\left(\frac{r_{2, \max }}{r_{1, \max }}\right)^{1 / 2},
$$

where $q_{1}=q_{2}=11 / 6$, and we have made use of Eq. (30) in integrating from 0 to $m_{c}$. For a sufficiently broad size distribution for the satellitesimals, $I_{s p} \sim I_{c s}$ (see footnote [12]). Given that in order to satisfy the long timescale of formation of Callisto $\tau_{z 2} \sim 10^{-5}, I_{s p}$ is likely to be small as indicated above. We can see this if we examine a case such that $\tau_{z 2}=1$, which means $r_{c}=r_{2, \max }$. Then all planetesimals with $r \leq r_{2, \max }$ could be captured into the disk. However, this condition is quite restrictive. For instance, a disk with the mass of the Galilean satellites out to $\sim 30 R_{J}$ has $\sigma_{2} \sim 10^{3} \mathrm{~g} \mathrm{~cm}^{-2}$, which implies $r_{2, \max } \sim 1-10 \mathrm{~m}$. Even if such a disk could be produced, the formation time for the satellites would be short, which would be inconsistent with Callisto's partially differentiated state.

Now we consider the mass delivery to the circumplanetary disk due to the interaction of planetesimal fragments with larger satellitesimals. The expected number of collisions of a disk crossing planetesimal of mass $m$ with satellitesimals of mass $m^{\prime}>m$ is

$$
\begin{aligned}
& \mathcal{E}\left(m ; m^{\prime}>m\right)=\int_{m}^{m_{2, \text { max }}} \xi \pi r^{\prime 2} H_{2} n_{2}\left(m^{\prime}\right) d m^{\prime} \simeq \\
& \left(\frac{3}{4 \pi \rho_{s}}\right)^{2 / 3} \pi \sigma_{2} \frac{2-q_{2}}{q_{2}-5 / 3} \frac{m^{5 / 3-q_{2}}}{m_{2, \max }^{2-q_{2}}}\left[1-\left(\frac{m}{m_{2, \max }}\right)^{q_{2}-5 / 3}\right],
\end{aligned}
$$


which is Eq. (11) of Safronov et al. (1986), where we have corrected an error in one of the exponents in that expression. Thus, the frequency of interaction of bodies with $m<m_{2, \max }$ is proportional to $m^{5 / 3-q_{2}}$ (Ruskol 1975). Integrating from $m_{2, \min }$ to $m_{2, \max }$ we find the three cases of interest for the mass inflow rate of planetesimals colliding with satellitesimals of larger mass

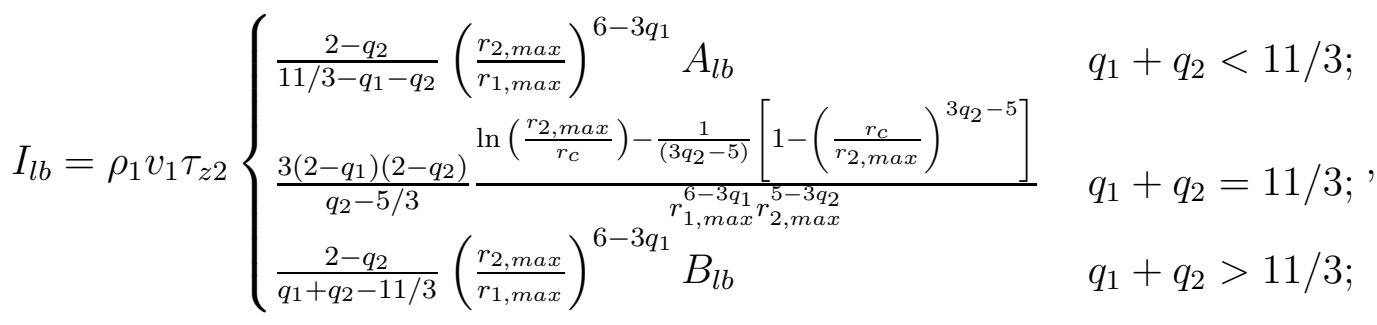

$$
\begin{aligned}
& A_{l b}=1-\frac{2-q_{1}}{q_{2}-5 / 3}\left(\frac{r_{c}}{r_{2, \max }}\right)^{11-3\left(q_{1}+q_{2}\right)}+\frac{11 / 3-q_{1}-q_{2}}{q_{2}-5 / 3}\left(\frac{r_{c}}{r_{2, \max }}\right)^{6-3 q_{1}} \\
& B_{l b}=\frac{2-q_{1}}{q_{2}-5 / 3}\left(\frac{r_{2, \max }}{r_{c}}\right)^{3\left(q_{1}+q_{2}\right)-11}+\frac{q_{1}+q_{2}-11 / 3}{q_{2}-5 / 3}\left(\frac{r_{c}}{r_{2, \max }}\right)^{6-3 q_{1}}-1
\end{aligned}
$$

where the lower limit of integration has been taken to be $r_{c}$. For our chosen values of $q_{1}=q_{2}=11 / 6$, the inflow rate due to larger bodies is

$$
I_{l b} \simeq \rho_{1} v_{1} \tau_{z 2}\left(\frac{r_{2, \max }}{r_{1, \max }}\right)^{1 / 2}\left[\tau_{z 2}+\ln \tau_{z 2}^{-1}-1\right],
$$

so that the total inflow rate into the circumplanetary disk for $q_{1}=q_{2}=11 / 6$ is

$$
I=I_{s p}+I_{l b} \approx \rho_{1} v_{1} \tau_{z 2}\left(\frac{r_{2, \max }}{r_{1, \max }}\right)^{1 / 2}\left[\tau_{z 2}-\ln \tau_{z 2}\right] .
$$

We see from this expression that for a low optical depth disk the mass delivery of incoming planetesimals by larger satellitesimals is more efficient than delivery by smaller or comparable sized bodies, as already mentioned. Note that in Eq. (36), the second term becomes zero for values of $\tau_{z 2} \geq 1$ since this corresponds to the situation in which $r_{c} \sim r_{2, \max }$ (i.e., all bodies smaller than $r_{2, \max }$ are captured into the disk).

The mass accretion rate obtained by integrating Eq. (36) over the disk area is

$$
\begin{aligned}
\dot{M}= & 2 \pi \int_{R_{0}}^{R_{D}} I(R) p(R) R d R \approx \\
& \frac{8 \pi \sigma_{1}}{P_{1}}\left(\frac{r_{2}}{r_{1}}\right)^{1 / 2} \int_{R_{0}}^{R_{D}} \tau_{z 2}\left[\tau_{z 2}-\ln \tau_{z 2}\right] p(R) R d R
\end{aligned}
$$


where $p(R)$ is given by Eq. (13), $l \equiv R / R_{P}$, and $r_{2}$ and $r_{1}$ are the satellitesimal and planetesimal size distribution cutoffs. We have expressed the quantity $\rho_{1} v_{1}$ in terms of the surface density of solids in the nebula $\sigma_{1}$, and the planetary period $P_{1}$.

We can now obtain solutions for the mass captured by the circumplanetary disk assuming that the mass delivery rate to the disk is balanced by the mass removal rate to the inner disk (and used to form close-in, prograde regular satellites). The mass removal rate is given by $\dot{M} \sim M_{s w} / \tau_{\text {coll }}$, where $M_{s w}$ is the steady state mass of the satellitesimal swarm (which excludes satellite embryos), and the collisional time is $\tau_{\text {coll }} \sim P_{2} / \tau_{z 2}$. We choose the characteristic orbital period in the disk to be $P_{2} \sim 1$ year, which is the orbital period at $R \sim R_{H} / 4$ (where the maximum of $p(R) \times R$ is located for $\left.\theta_{1} \sim 75\right)$. Given that the total mass to be delivered in the satellite's accretion time $\tau_{a c c}$ is $M \sim 10^{26} \mathrm{~g}$, we must have $M=M_{s w}+\dot{M} \times \tau_{a c c}$. This simply means that the disk mass must satisfy $M_{s w}=M /\left(1+N_{c}\right)$, where $N_{c}=\tau_{a c c} / \tau_{\text {coll }}$ is the number of collisional cycles. We can also write this equation in the form

$$
\dot{M} \tau_{a c c}-\frac{N_{c}}{1+N_{c}} M=0
$$

which allows us to express $r_{2}, \tau_{z 2}$, and $\sigma_{2}$ in terms of $N_{c}$, so that equations (37-38) can be solved by iteration as functions of $N_{c}$, for specific choices of the solar nebula parameters $r_{1}, \theta_{1}$ and $\sigma_{1}$. Here we assume that the optical depth and solids surface density of the circumplanetary disk are constant not only in time but with position as well.

Figures 1 and 2 correspond to a case such that the satellite accretion time (which is set by the planetesimal feeding time) is $\tau_{a c c}=10^{6}$ years, the solids surface density in the solar nebula is ten times MMSN, and the Safronov parameter $\theta_{1}=4\left(v_{10}=21\right.$ $\mathrm{km} \mathrm{s}^{-1}$ ). In Figure 1 we plot the satellitesimal cutoff size $r_{2}$ (solid line) and surface density $\sigma_{2}$ (dotted line) as a function of $r_{1}$ that satisfy the system constraints. In this case solutions for which $N_{c} \sim 1-100$ correspond to the range of $r_{1}$ shown. Larger values of $r_{2}$ correspond to larger $\tau_{\text {coll }}$ (smaller $\tau_{z 2}$ ) and hence fewer collisional cycles, as indicated in Figure 1 and by the dotted curve in Figure 2. The solids surface density $\sigma_{2}$ changes only by an order of magnitude over the same range in which the satellitesimal maximum cutoff size varies by a factor of $\sim 1000$. The greater variation of $r_{2}$ compared to $\sigma_{2}$ is a result of the of the stronger dependence of $r_{2} \propto N_{c}^{-1}\left(1+N_{c}\right)^{-1}$ compared to $\sigma_{2} \propto\left(1+N_{c}\right)^{-1}$. It should be pointed out that there are solutions for $N_{c} \sim 1$. This corresponds to a case in which the steady state disk mass is $\sim 0.5$ the mass the Galilean satellites. It is unclear, however, whether the required size of $r_{2} \sim 10 \mathrm{~km}$ (for $\theta_{1}=4$ ) is too large to be realistic. We do not attempt to model their size distribution, but a population of such large satellitesimals may be difficult to maintain given fragmentation from hypervelocity collisions from Rochelobe interlopers. We discuss this point in more detail at the end of this section. Here we note that the range of possible values of $r_{2}$ is quite broad, so that an independent constraint for this quantity is essential.

Next we do a case in which $\sigma_{1}$ is two times the solids MMSN, $\tau_{a c c}=10^{6}$ years, 


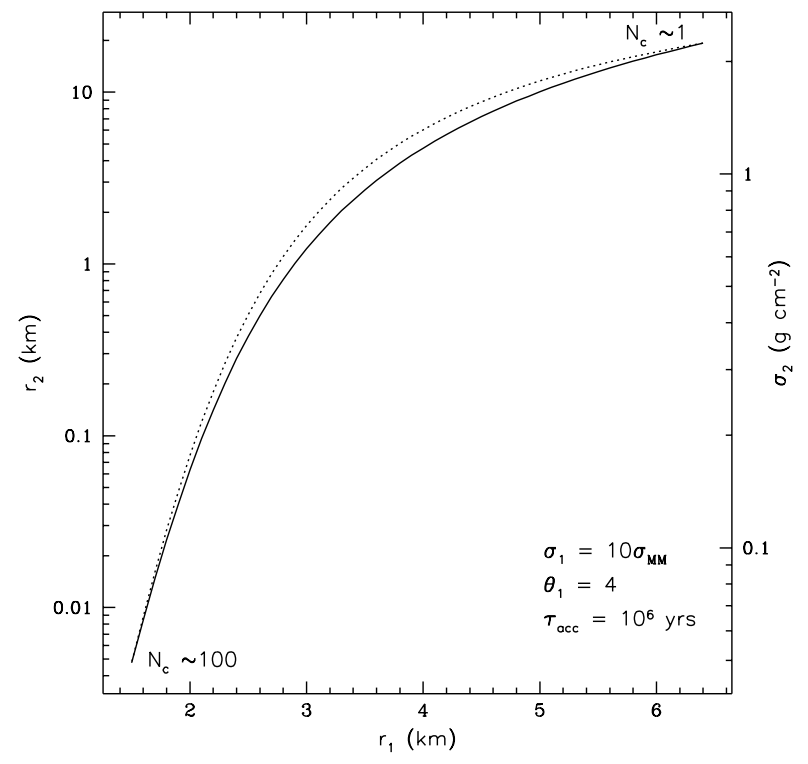

Figure 1: Plot of the satellitesimal cutoff size $r_{2}$ (solids line) and solids surface density $\sigma_{2}$ (dotted line) as a function of $r_{1}$ for a case in which the solar nebula is ten times minimum mass of solids, a planetesimal feeding time $\tau_{a c c}=10^{6}$ years, and $\theta_{1}=4$. The extremes of $N_{c}$ are indicated.

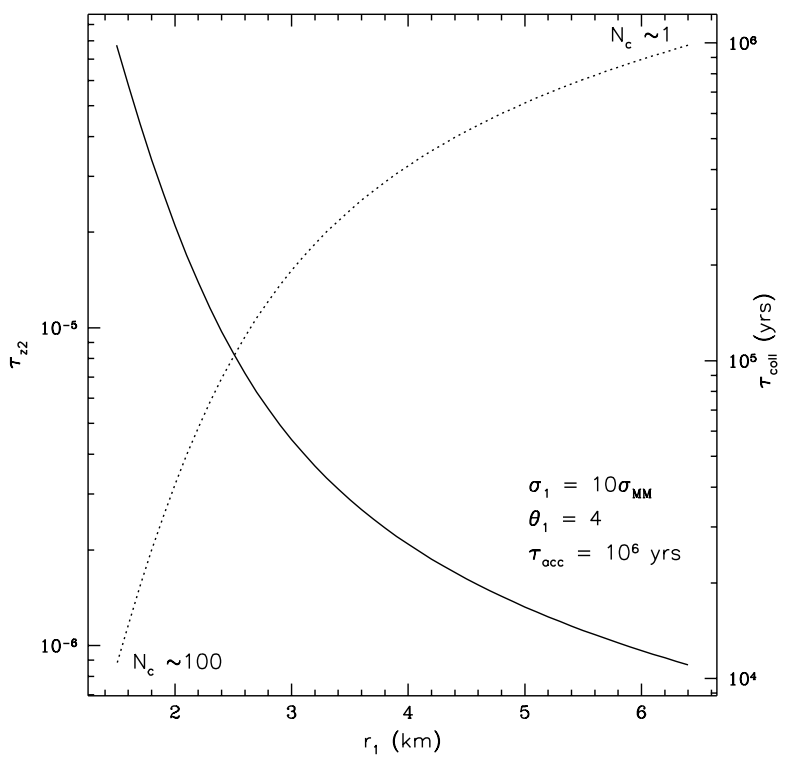

Figure 2: Plot of the optical depth $\tau_{z 2}$ (solid line) and collisional time $\tau_{\text {coll }}$ (dotted line) as a function of $r_{1}$ for a case in which the solar nebula is assumed to have ten times the minimum mass of solids, a planetesimal feeding time of $\tau_{a c c}=10^{6}$ years, and $\theta_{1}=4$. The extremes of $N_{c}$ are indicated. 


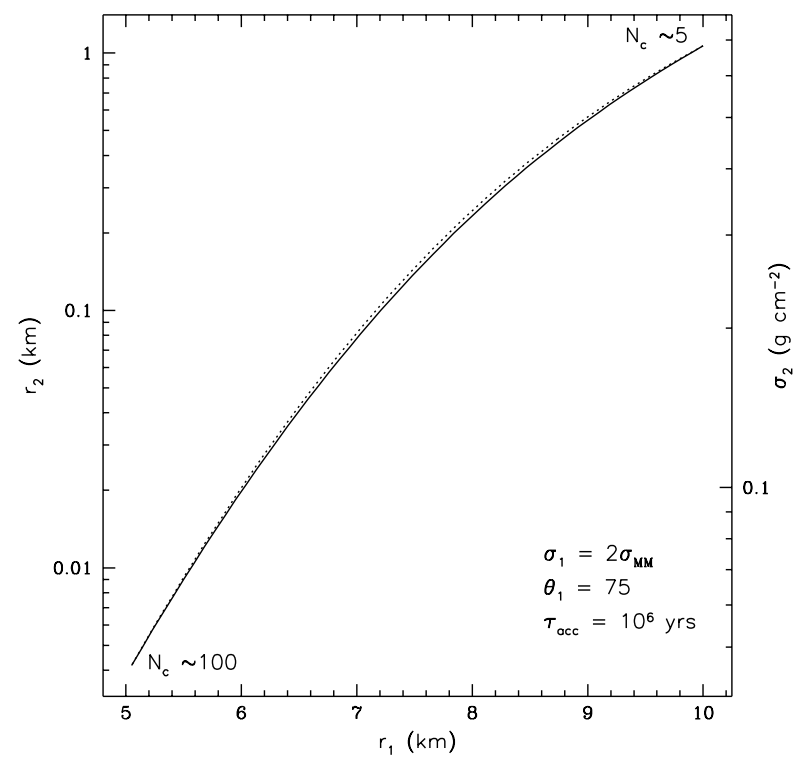

Figure 3: Same as Figure 1, except that the solids surface density in the solar nebula is two times minimum mass and $\theta_{1}=75$ (characteristic of a colder planetesimal population). In this case, the range of values for $r_{1}$ are somewhat broader, but significantly narrower for $r_{2}$.

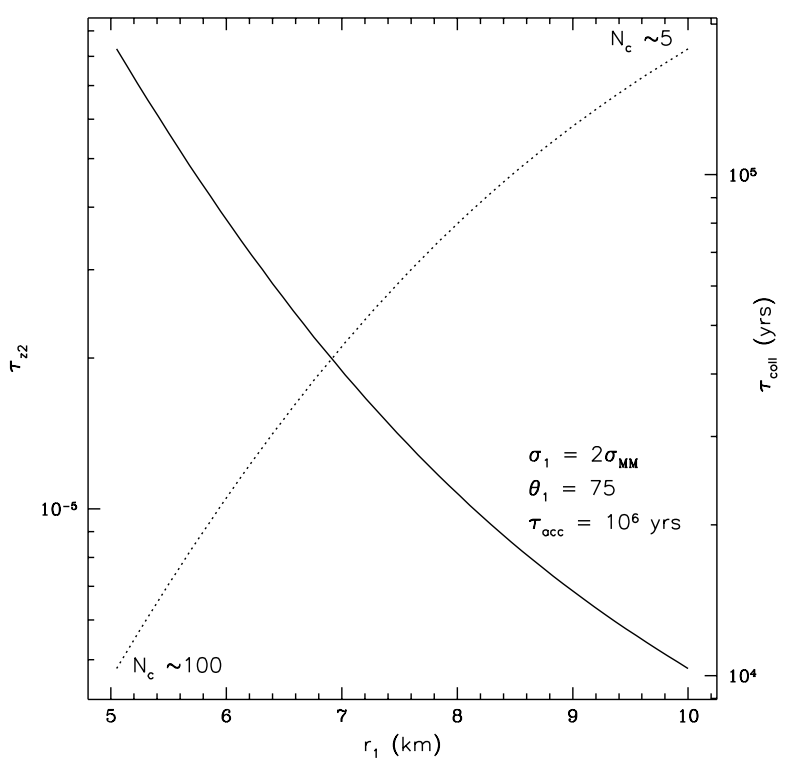

Figure 4: Same as Figure 2, except that the solids surface density in the solar nebula is two times minimum mass and $\theta_{1}=75$. About $N_{c} \sim 5$ collisional cycles are needed to complete the accretion of the satellites in the case of $r_{1}=10 \mathrm{~km}$ and $r_{2}=1 \mathrm{~km}$. 


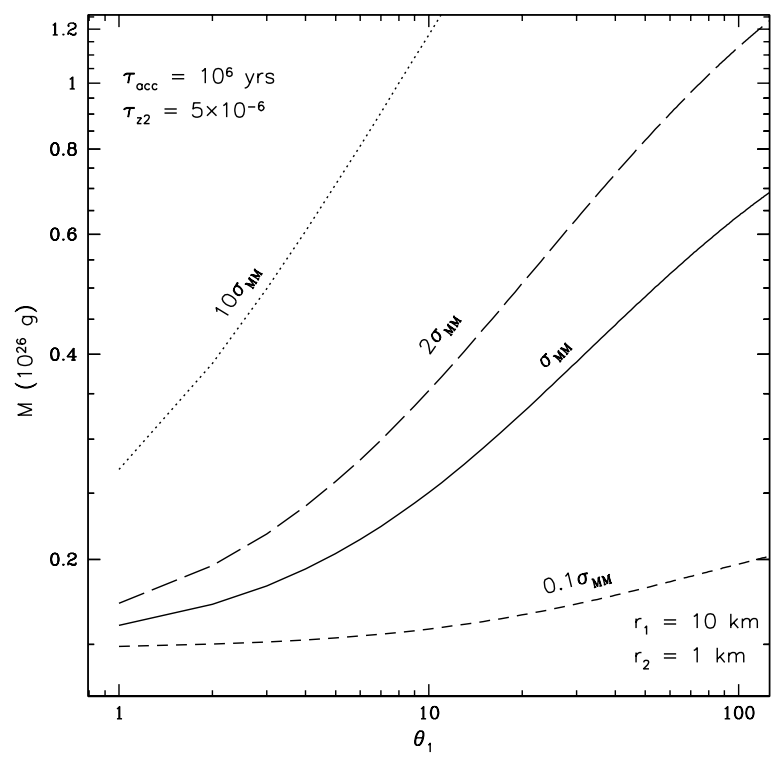

Figure 5: Plot of the mass accreted over $10^{6}$ years as a function of the planetesimal Safronov parameter $\theta_{1}$. For these curves $\tau_{z 2} \sim 5 \times 10^{-6}, r_{1}=10 \mathrm{~km}$, and $r_{2}=1 \mathrm{~km}$.

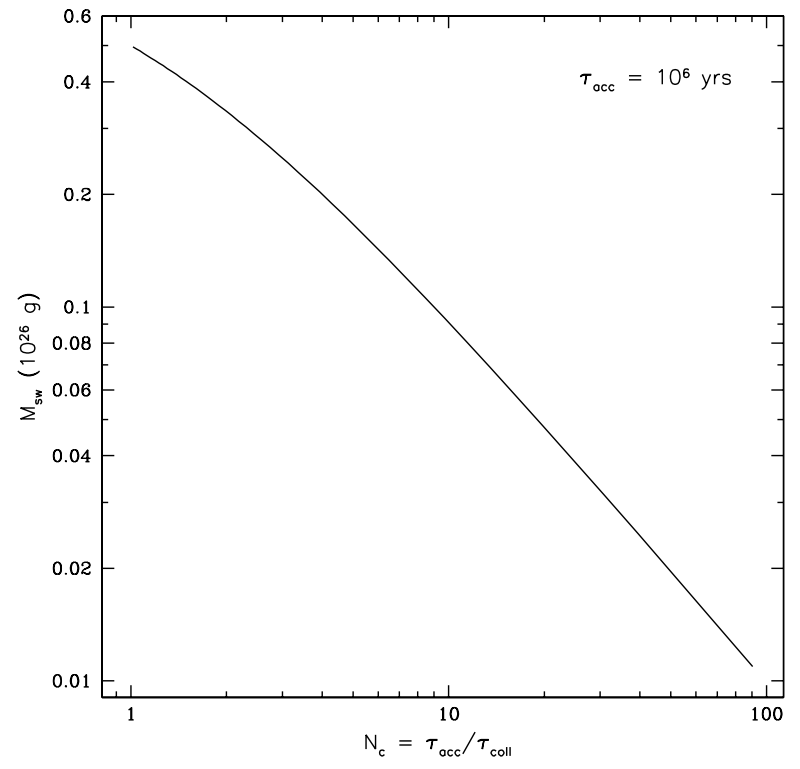

Figure 6: The steady-state disk mass $M_{s w}$ as a function of collisional cycles $N_{c}$ for $\tau_{a c c}=10^{6}$ years. The mass $M_{s w}$ is removed to the inner disk each $\tau_{\text {coll }}$ so this mass must be replenished each cycle. 
and we assume $\theta_{1}=75\left(v_{10}=5 \mathrm{~km} \mathrm{~s}^{-1}\right)$, implying a fairly "cold" population of heliocentric objects (Figures 3 and 4 ). We find solutions for $r_{1} \sim 5-10 \mathrm{~km}$, which correspond to $N_{c}$ in the range $5-100$. In particular, a solution exists where the satellitesimal cutoff size is $r_{1}=1 \mathrm{~km}$ for a planetesimal cutoff size of $r_{1}=10 \mathrm{~km}$. In this case, $\tau_{z 2} \sim 5 \times 10^{-6}$ implying that timescale for the collisional cycle is $\tau_{\text {coll }} \sim$ $2 \times 10^{5}$ years, so that $N_{c} \sim 5$ collisional cycles are required to complete the formation of the satellites. We note that the range of variation of both $r_{2}$ and $\sigma_{2}$ is significantly smaller for this case, which suggests that large $\theta_{1}$ cases may be easier to constrain.

To understand better the effect of the velocity dispersion of the heliocentric planetesimal population on the mass delivery rate, we plot in Figure 5 the total mass delivered to the circumplanetary disk over and accretional time of $\tau_{a c c}=10^{6}$ years as a function of the Safronov parameter $\theta_{1}$. In this case we fix $r_{1}$ and $r_{2}$ at 10 and $1 \mathrm{~km}$ respectively. All of the cases presented assume that $\tau_{z 2}=5 \times 10^{-6}$ so that $N_{c}=5$. The solid curve corresponds to a solids MMSN $\left(\sigma_{\mathrm{MM}}\right)$, the long dashed line corresponds to a case of $\sigma_{1}=2 \sigma_{\mathrm{MM}}$, the dotted line to $\sigma_{1}=10 \sigma_{\mathrm{MM}}$, and the short-dashed line to $\sigma_{1}=0.1 \sigma_{\mathrm{MM}}$. From this plot we can see that for a choice of $\theta_{1}=4$ to have a solution for $r_{1}=10 \mathrm{~km}$ one would require a case with greater nebula solid content than ten times MMSN (cf. Figure 1). Conversely, one could have a slightly colder population $\left(\theta_{1} \sim 8\right)$ that satisfy the constraints for the case of ten times MMSN.

Finally, in Figure 6 we plot the mass captured by the circumplanetary disk in one collision time for $\tau_{a c c}=10^{6}$ years as a function of collisional cycles $N_{c}$. This mass simply corresponds to the steady-state disk mass $M_{s w}$ as it should. All prior solutions for a range of $r_{1}$ lie on this curve. For instance, the case for twice MMSN and $r_{1}=10$ $\mathrm{km}$ for which $N_{c} \sim 5$ collisional cycles implies that $M \sim 0.2$ Galilean masses are captured per collision time $\tau_{\text {coll }} \sim 2 \times 10^{5}$ years (corresponding to $\dot{M} \sim 5 \times 10^{20}$

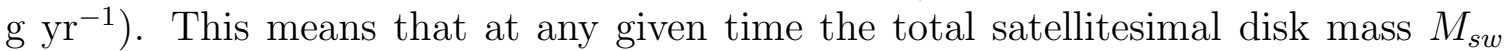
(excluding embryos) is $\sim 20 \%$ of the total mass of the satellites.

An important caveat is that all these cases use accretion times that are significantly longer that feeding zone clearing timescale, which means that we are assuming some mechanism to replenish it. It is also important to point out that at these late times modeling of the planetesimal mass spectrum would involve two regimes with different power law exponents, and a break location that changes with time (see, e.g., Kenyon and Bromley 2004, Pan and Sari 2004). Here and in the next section we consider small values for $r_{1} \sim 1 \mathrm{~km}$ simply to indicate that following giant planet formation a significant fraction of the planetesimal mass in the feeding zone of the giant planet may cascade back down to small sizes. Finally, one would still need to solve self-consistently for the size of $r_{2}$. This size might be set by a competition between the growth time of satellitesimals versus the time it takes for catastrophic collisions with incoming planetesimals to disrupt them. The growth time of $r_{2}$ is $\sim \tau_{\text {coll }}$ which may be, as for the cases in Figure 3 and 4 , quite long $\sim 10^{5}$ years. We can estimate the lifetime of a satellitesimal of size $r_{2}$ as the time it takes for a bigger planetesimal to collide with it. Given a planetesimal size distribution with upper cutoff of $r_{1}=10$ $\mathrm{km}$ and power law exponent $q_{1}=11 / 6$, the time for a large planetesimal to shatter 


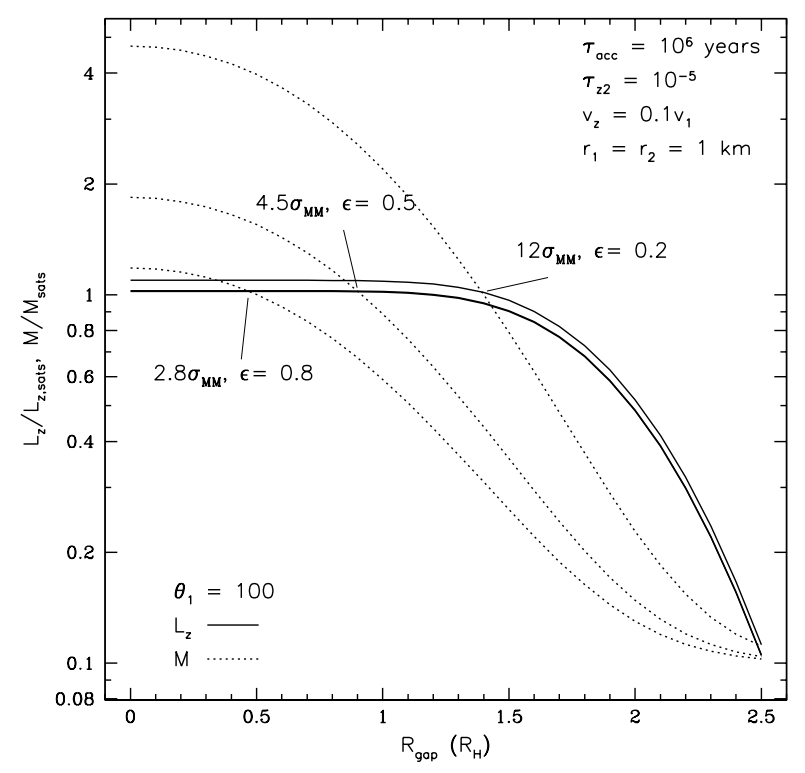

Figure 7: Mass (dotted lines) and angular momentum (solid lines) in units of the mass and angular momentum of the Galilean satellites $\left(M_{\text {sats }} \sim 4 \times 10^{26} \mathrm{~g}, L_{z \text {,sats }} \sim 4 \times 10^{43}\right.$ $\mathrm{g} \mathrm{cm}^{2} \mathrm{~s}^{-1}$ ) accreted by the circumplanetary disk in $\tau_{a c c}=10^{6}$ years as a function of the gap size $R_{\text {gap }}=\sqrt{\ell_{0} / \Omega_{1}}$. For this case $N_{c}=10$, implying a steady-state disk mass is $M_{s w}=0.1 M_{\text {sats }}$. The proper budget of mass and angular momentum to account for the Galilean satellites is achieved where the solid and the dotted lines intersect at $L_{z} / L_{z, \text { sats }}=M / M_{\text {sats }}=1$. For example, for the case of $\sigma_{1}=4.5 \sigma_{\mathrm{MM}}, R_{g a p} \sim 1 R_{H}$.

$\mathrm{a} \sim 1 \mathrm{~km}$ satellitesimal can be estimated to be $\tau_{\text {frag }} \sim 10^{-3} P_{1} \rho_{s}\left(r_{1} r_{2}\right)^{1 / 2} / \sigma_{1} \sim 10^{3}$ years for a solids MMSN. This is $\sim 100$ times faster than its growth time, suggesting that the cutoff in $r_{2}$ should be smaller than $1 \mathrm{~km}$, and invalidating the assumptions used to obtain model solutions. This result is a direct consequence of the model constraints coupled with a small capture probability $p(R)<<1$. However, it may be possible to circumvent this problem by speeding up the accretion of satellitesimals using circumplanetary debris, and increasing the capture probability by using a large value of the Safronov parameter $\theta_{1}$. It is also possible that a different process, such as the capture of planetesimal fragments, is responsible for setting the satellitesimal cutoff size. However, we leave these complications for further work.

\subsection{Angular Momentum Delivery}

In this section we show that it may be possible to deliver enough angular momentum to the circumplanetary disk to explain the observed angular momentum budget of the regular satellite systems. At first this might appear to be a problem given that the circumplanetary disk is composed of both prograde and retrograde satellitesimals with small net angular momentum. However, as the giant planet clears planetesimals 
from its feeding zone on a timescale comparable to the satellite formation timescale, inhomogeneities in the planetesimal disk can pump up the angular momentum delivered to the circumplanetary disk (a point first made by Lissauer and Kary 1991 in the context of planetary spins), which may account for the observed radial extent of the satellite systems provided that most of the solids that make up the satellites originate from the outer regions of the giant planet's feeding zone.

Here we assume that the bulk of the angular momentum that goes into the regular satellites arrives late as the planet clears its feeding zone, and that the planetesimal population at this time is composed of small particles $r_{1}<r_{2} \lesssim 1 \mathrm{~km}$ with a velocity dispersion characteristic of a fairly cold population $\left(\theta_{1} \sim 100\right.$, but below we still use the "high" dispersion limit) with lower inclinations than eccentricities $\left(v_{z}=0.1 v_{1}\right)$. This implies that the capture probability $p(R) \lesssim 1$. Furthermore, we assume that on average a free-bound collision deposits a significant fraction $\epsilon \sim 0.5$ of the angular momentum of the interloper. This is because colliding planetesimals may deposit angular momentum in the circumplanetary disk even if they (or their fragments) are not captured ${ }^{13}$.

We provide an estimate for the amount of angular momentum delivered to the circumplanetary disk using a method which is related to planetary spin calculations (e.g., Lissauer and Kary 1991; Dones and Tremaine 1993, hereafter DT93). We calculate the rate of accretion of mass and angular momentum by a (2-D) disk of satellitesimals. Our simplifying assumptions are that the planet is on a circular orbit, that incoming planetesimals do not interact with each other, and that $\sigma_{1}$ is uniform over the planet's (undepleted portion of the) feeding zone. Incoming particle velocities increase as they approach the planet. This prevents us from considering significantly larger values than $\theta_{1} \sim 100$, and leads us to adjust the velocity dispersion using $v_{1}=\sqrt{v_{10}^{2}+v_{e}^{2}}$, with $v_{10}$ and $v_{e}$ as previously defined. ${ }^{14}$ In addition, the giant planet's mass and the properties of the circumplanetary disk are assumed to be constant. With these assumptions, the problem can be cast in terms of two dimensionless parameters

$$
b \equiv \frac{R}{R_{H}}, \quad s \equiv \frac{v_{1}}{\Omega_{1} R_{H}}
$$

where $b$ is the ratio of radial distance in the circumplanetary disk to the Hill radius, and $s$ measures the importance of the differential rotation of the circumsolar disk relative to the velocities of incoming planetesimals (DT93). The parameter $b$ is a measure of the importance of the planet's gravity relative to that of the Sun at $R$ in the satellitesimal disk. For our problem, $\theta_{1} \gtrsim 100$ implies $v_{1} \lesssim 6 \mathrm{~km} \mathrm{~s}^{-1}$ and thus $s \lesssim 6$. At $R_{H} / 4$ (where $p(R) \times R$ peaks for $\theta_{1} \sim 100$ ), $b / s \lesssim 1$ and $b s^{2} \gtrsim 1$, implying

\footnotetext{
${ }^{13}$ One might consider the case $\epsilon=p(R)$ as a lower bound on the angular momentum deposition efficiency. This would require that a planetesimal (or its fragments) be captured in order to deliver its angular momentum to the disk. For our choice of parameters $p(R) \lesssim \epsilon$.

${ }^{14}$ It should be noted that the satellitesimals Keplerian velocity is not much smaller than $v_{1}$. This could affect the calculation for the net angular momentum deposition. We leave investigation of this complication for further work.
} 


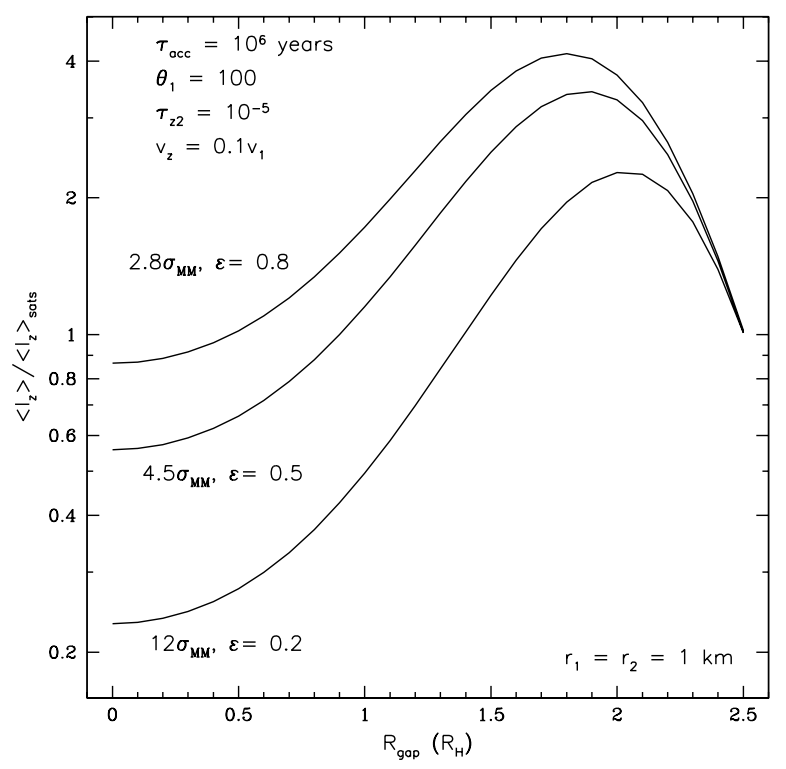

Figure 8: Specific angular momentum delivered in units of the specific angular momentum of the Galilean satellites $\left\langle\ell_{z}\right\rangle_{\text {sats }} \sim 1 \times 10^{17} \mathrm{~cm}^{2} \mathrm{~s}^{-1}$ as a function of gap size $R_{\text {gap }}=\sqrt{\ell_{0} / \Omega_{1}}$. These curves correspond to cases such that $\left\langle\ell_{z}\right\rangle /\left\langle\ell_{z}\right\rangle_{\text {sats }}=1$ matches the mass and angular momentum budget of the Galilean satellite system.

we are in a regime in which dispersion is still high and gravity is somewhat important.

The accretion of mass and angular momentum by the circumplanetary disk can be described by a 3 -D distribution $\Phi\left(R, \ell_{z}\right)$, where $\Phi\left(R, \ell_{z}\right) d \ell_{z}$ is the collisional inflow rate per unit area of mass with specific angular momentum $\ell_{z}$ in the range $\ell_{z}$ and $\ell_{z}+d \ell_{z}$. For our problem, we have

$$
\begin{array}{r}
\Phi\left(R, \ell_{z}\right)=2 \int_{0}^{2 \pi} d \phi \int_{-\infty}^{\infty} d \dot{x} \int_{-\infty}^{\infty} d \dot{y} \int_{-\infty}^{\infty} d \dot{z} \tau(\vartheta) \times \\
f_{0}\left(x, y, z \rightarrow 0^{+}, \dot{x}, \dot{y}, \dot{z}\right) \delta\left(\ell_{z}-x \dot{y}+y \dot{x}-\Omega_{1} R^{2}\right)|\dot{z}| H(-\dot{z}),
\end{array}
$$

where the azimuthal angle $\phi$ is measured from the origin of the system centered on the planet such that $(x, y)=R(\cos \phi, \sin \phi)$, the $x-y$ plane is the planetary orbit plane (which coincides with the circumplanetary disk plane), the $x$-axis points radially outward, the $y$-axis points in the direction of orbital motion, $\delta$ is the Dirac delta function, and $H(-\dot{z})$ is the Heaviside step function, which selects only planetesimals with velocities directed towards the disk plane. The notation $z \rightarrow 0^{+}$is meant to indicate that we are approaching the disk plane from above $(z>0)$. The probability of a free-bound collision in crossing the disk $\tau(\vartheta)$ is given by 


$$
\tau(\vartheta)=\frac{\tau_{z 2}}{|\sin \vartheta|}=\tau_{z 2}\left(\frac{v_{r}^{2}+v_{\phi}^{2}+\dot{z}^{2}}{\dot{z}^{2}}\right)^{1 / 2} \sim \tau_{z 2} \frac{\left|v_{r}\right|}{|\dot{z}|},
$$

where $v_{r}=\dot{x} \cos \phi+\dot{y} \sin \phi$ is the radial velocity, $v_{\phi}$ is the azimuthal velocity, and we have assumed that the planetesimal radial velocities dominate their motion (which implies a shallow angle of entry). In Eq. (40), $f_{0}$ is a Schwarzchild distribution function, describing the mass of a uniform disk of particles in a three-dimensional phase-space volume element $d x d y d z d \dot{x} d \dot{y} d \dot{z}$.

$$
\begin{gathered}
f_{0}(x, y, z, \dot{x}, \dot{y}, \dot{z})= \\
\frac{\rho_{0}}{(2 \pi)^{3 / 2} v_{1}^{2}\left(1-\frac{1}{2} \Gamma\right)^{1 / 2} v_{z}} \exp \left[-\frac{\Omega_{1}^{2} z^{2}}{2 v_{z}^{2}}-\frac{\dot{x}^{2}}{2 v_{1}^{2}}-\frac{\left(\dot{y}+\Gamma \Omega_{1} x\right)^{2}}{2 v_{1}^{2}\left(1-\frac{1}{2} \Gamma\right)}-\frac{\dot{z}^{2}}{2 v_{z}^{2}}\right],
\end{gathered}
$$

which may apply sufficiently far from the planet. In this expression, $\rho_{0}=\sqrt{2 \pi} \sigma_{1} / P_{1} v_{z}$ is the midplane planetesimal volume density, and we have assumed that the $x$ component of the velocity dispersion $v_{x}=v_{1}$, and $v_{y}=v_{1}\left(1-\frac{1}{2} \Gamma\right)^{1 / 2}$, where $\Gamma=$ $-(d \ln \Omega / d \ln R)=3 / 2$ for a Keplerian disk.

Integrating Eq. (40) over $\dot{z}$, defining the velocities $(\dot{x}, \dot{y}) \equiv(v \cos \psi, v \sin \psi)$, and replacing the azimuthal angle $\phi$ with $\chi=\pi+\phi-\psi$ so that $\left|v_{r}\right|=-v \cos \chi$, we can write in polar coordinates (DT93)

$$
\begin{aligned}
\Phi\left(R, \ell_{z}\right)= & \frac{2 \sigma_{1} \tau_{z 2}}{\left[2 \pi\left(1-\frac{1}{2} \Gamma\right)\right]^{1 / 2} v_{1}^{2} v_{z} P_{1}} \int_{0}^{2 \pi} d \psi \int_{-\pi / 2}^{\pi / 2} \cos \chi d \chi \\
& \int_{\left|\ell_{z}-\Omega_{1} R^{2}\right| / R}^{\infty} v^{2} e^{-\frac{1}{2} \Psi} \delta\left(\ell_{z}-R v \sin \chi-\Omega_{1} R^{2}\right) d v
\end{aligned}
$$

and

$$
\Psi \equiv \frac{v^{2} \cos ^{2} \psi}{v_{1}^{2}}+\frac{\left[v \sin \psi-\Gamma \Omega_{1} R \cos (\chi+\psi)\right]^{2}}{v_{1}^{2}\left(1-\frac{1}{2} \Gamma\right)} .
$$

Generally, if the velocity dispersion is large enough or for small $b$, the quantity $b / s<<1$, and Eq. (43) can be simplified by expanding Eq. (44) to first order in $b / s$. However, for our problem $b / s \gtrsim 0.1$ so that we expand Eq. (44) to fourth order to obtain a better estimate. Carrying out the expansion and integrating over $\chi$ and $v$ (see Appendix B)

$$
\begin{array}{r}
\Phi(R, \alpha)=\frac{2 \sigma_{1} \tau_{z 2}}{R\left[2 \pi\left(1-\frac{1}{2} \Gamma\right)\right]^{1 / 2} v_{z} P_{1}} \int_{0}^{2 \pi} \frac{d \psi}{g(\psi)}\left[\left(K_{0}(g)+K_{1}(g) \alpha+K_{2}(g) \alpha^{2}+\right.\right. \\
\left.K_{3}(g) \alpha^{3}+K_{4}(g) \alpha^{4}\right) e^{-g \alpha^{2}}+\left(K_{5}(g) \alpha^{2}+K_{6}(g) \alpha^{3}+\right. \\
\left.\left.K_{7}(g) \alpha^{4}\right) E_{1}\left(g \alpha^{2}\right)\right]
\end{array}
$$


where the $K_{i}$ are functions of the angle $\psi, \Gamma$, and $b / s$. Here $\alpha=\left(\ell_{z}-\Omega_{1} R^{2}\right) / \sqrt{2} v_{1} R$, $E_{1}$ is the exponential integral of order 1 , and

$$
g(\psi)=\cos ^{2} \psi+\frac{\sin ^{2} \psi}{1-\frac{1}{2} \Gamma} .
$$

To obtain the mass capture rate of planetesimals with specific angular momentum $\left|\ell_{z}\right| \geq \ell_{0}$ we define the mass inflow collision rate per unit area $\mathcal{I}\left(R, \ell_{0}\right)$

$$
\begin{aligned}
\mathcal{I}\left(R, \ell_{0}\right)=\frac{1}{2 \pi} \int_{\ell_{0}}^{\infty} \Phi\left(R, \ell_{z}\right) d \ell_{z} & +\frac{1}{2 \pi} \int_{-\infty}^{-\ell_{0}} \Phi\left(R, \ell_{z}\right) d \ell_{z}= \\
& \frac{\sqrt{2} v_{1} R}{2 \pi}\left\{\int_{\alpha_{0}}^{\infty} \Phi(R, \alpha) d \alpha+\int_{\alpha_{0}^{\prime}}^{\infty} \Phi\left(R, \alpha^{\prime}\right) d \alpha^{\prime}\right\}^{\prime}
\end{aligned}
$$

where $\Phi\left(R, \alpha^{\prime}\right)$ is identical to Eq. (45), except with the replacements $K_{1} \rightarrow-K_{1}$, $K_{3} \rightarrow-K_{3}, K_{6} \rightarrow-K_{6}$, and $\alpha^{\prime}=\left(\ell_{z}+\Omega_{1} R^{2}\right) / \sqrt{2} v_{1} R$. Integrating over the disk area, we get the mass capture rate

$$
\begin{aligned}
& \dot{M}\left(\ell_{0}\right)=2 \pi \int_{R_{0}}^{R_{D}} \mathcal{I}\left(R, \ell_{0}\right) p(R) R d R= \\
& \left.\quad \frac{2 \sigma_{1} \tau_{z 2}}{\left(1-\frac{1}{2} \Gamma\right)^{1 / 2} P_{1}} \int_{R_{0}}^{R_{D}} \frac{v_{1}}{v_{z}} p(R) R d R \int_{0}^{2 \pi} \frac{d \psi}{[g(\psi)]^{3 / 2}} \sum_{i=0}^{7} \mathcal{M}_{i}\left(g(\psi), R, \ell_{0}\right)\right)
\end{aligned}
$$

where $p(R)$ is given by Eq. (13), and the $\mathcal{M}_{i}$ are given in Appendix B. The total mass delivered to the circumplanetary disk is then given by $M=M_{s w}+\dot{M} \tau_{a c c}$, where as before $M_{s w}$ is the steady-state circumplanetary disk mass (excluding satellite embryos).

The angular momentum rate delivered to the circumplanetary disk $\dot{L}_{z}$ can be calculated from the first moment of the distribution $\Phi\left(R, \ell_{z}\right)$. The angular momentum delivery rate per unit area $\mathcal{I}_{L}\left(R, \ell_{0}\right)$ assuming perfect deposition efficiency is

$$
\begin{gathered}
\mathcal{I}_{L}\left(R, \ell_{0}\right)=\frac{1}{2 \pi} \int_{\ell_{0}}^{\infty} \ell_{z} \Phi\left(R, \ell_{z}\right) d \ell_{z}+\frac{1}{2 \pi} \int_{-\infty}^{-\ell_{0}} \ell_{z} \Phi\left(R, \ell_{z}\right) d \ell_{z}= \\
\Omega_{1} R^{2} \mathcal{I}(R)+\frac{v_{1}^{2} R^{2}}{\pi}\left\{\int_{\alpha_{0}}^{\infty} \alpha \Phi(R, \alpha) d \alpha-\int_{\alpha_{0}^{\prime}}^{\infty} \alpha^{\prime} \Phi\left(R, \alpha^{\prime}\right) d \alpha^{\prime}\right\}^{\prime}
\end{gathered}
$$

which when integrated over the circumplanetary disk yields 


$$
\begin{aligned}
& \dot{L}_{z}\left(\ell_{0}\right)=2 \pi \int_{R_{0}}^{R_{D}} \epsilon \mathcal{I}_{L}\left(R, \ell_{0}\right) R d R=\frac{2 \epsilon \sigma_{1} \tau_{z 2} \Omega_{1}}{\left(1-\frac{1}{2} \Gamma\right)^{1 / 2} P_{1}} \int_{R_{0}}^{R_{D}} \frac{v_{1}}{v_{z}} R d R \times \\
& \left.\left.\left\{R^{2} \int_{0}^{2 \pi} \frac{d \psi}{[g(\psi)]^{3 / 2}} \sum_{i=0}^{7} \mathcal{M}_{i}\left(g(\psi), R, \ell_{0}\right)\right)+\frac{\sqrt{2} v_{1} R}{\Omega_{1}} \int_{0}^{2 \pi} \frac{d \psi}{[g(\psi)]^{3 / 2}} \sum_{i=0}^{7} \mathcal{L}_{i}\left(g(\psi), R, \ell_{0}\right)\right)\right\}
\end{aligned}
$$

where the $\mathcal{L}_{i}$ are given in Appendix $\mathrm{B}$. The total angular momentum delivered to the circumplanetary disk is then $L_{z}=L_{i}+\dot{L}_{z} \tau_{a c c}$, where $L_{i}$ is the initial angular momentum of the disk, which is taken to be small. The specific angular momentum is $\left\langle\ell_{z}\right\rangle=L_{z} / M$.

We integrate Eq. (48) and (50) over angle $\psi$ and radius using standard numerical techniques (note that, although $v_{1}$ varies with $R$, we held the ratio $v_{1} / v_{z}$ constant in these calculations). In Figure 7 we plot both the mass $M$ and $z$-component of angular momentum $L_{z}$ delivered to the circumplanetary disk over the planetesimal feeding time $\tau_{a c c}=10^{6}$ years as a function of the feeding zone gap-size $R_{\text {gap }}=\sqrt{\ell_{0} / \Omega_{1}}$ with $R_{0}=R_{P}$ and $R_{D}=R_{H} / 2$. It is important to note that this is a practical definition based on the observation that for $R_{\text {gap }}>2.5 R_{H}$ the mass inflow rate drops to nearly zero. Because in the high dispersion weak gravity limit large magnitude (planetesimals may deposit positive or negative) angular momentum contributions are not limited to the outer regions of the feeding zone of the giant planet, this calculation likely overestimates the increase in specific angular momentum as a gap is opened ${ }^{15}$. Here we are mainly interested in the relative behavior of the angular momentum curves to the mass curves. The mass and angular momentum are plotted so that unity corresponds to the total mass and angular momentum contained in the Galilean satellites, $M_{\text {sats }} \sim 4 \times 10^{26} \mathrm{~g}, L_{z \text {,sats }} \sim 4 \times 10^{43} \mathrm{~g} \mathrm{~cm}^{2} \mathrm{~s}^{-1}$, which yields $\left\langle\ell_{z}\right\rangle_{\text {sats }} \sim 1 \times 10^{17} \mathrm{~cm}^{2} \mathrm{~s}^{-1}$. In this figure we plot curves for combinations of surface density $\sigma_{1}$ and angular momentum deposition efficiency $\epsilon$ such that solutions matching the mass and angular momentum in the Galilean satellites were found for gap sizes $R_{\text {gap }} \sim 1 R_{H}$. This implies that for our chosen parameters the bulk of the solids that form the Galilean satellites can be delivered as the giant planet clears a gap in its feeding zone. This can work provided that a significant fraction of the mass of the solids is in planetesimal fragments with $r_{1} \lesssim r_{2}$ with low inclination orbits $v_{z}=0.1 v_{1}$, that collisions are efficient at depositing angular momentum $\epsilon \sim 0.5$, and that the nebula is enhanced in solids with respect to MMSN. Given a value for the satellitesimal size cutoff of $r_{2}=1 \mathrm{~km}$, we then have $\tau_{z 2} \sim 10^{-5}, \tau_{\text {coll }} \sim 10^{5}$ years (since the dynamical time in the satellitesimal disk is $\sim 1$ year), $N_{c} \sim 10, M_{s w} \sim 0.1 M_{\text {sats }}$ as before (which is the value the mass curves [dotted lines] approach as the gap size $R_{\text {gap }}$ encompasses the giant planet's feeding zone), and $L_{i}<0.1 L_{\text {sats }}$. We can also define the quantity

\footnotetext{
${ }^{15}$ Ohtsuki and Ida (1998) argue that such a boundary should be implemented in terms of the Jacobi constant.
} 


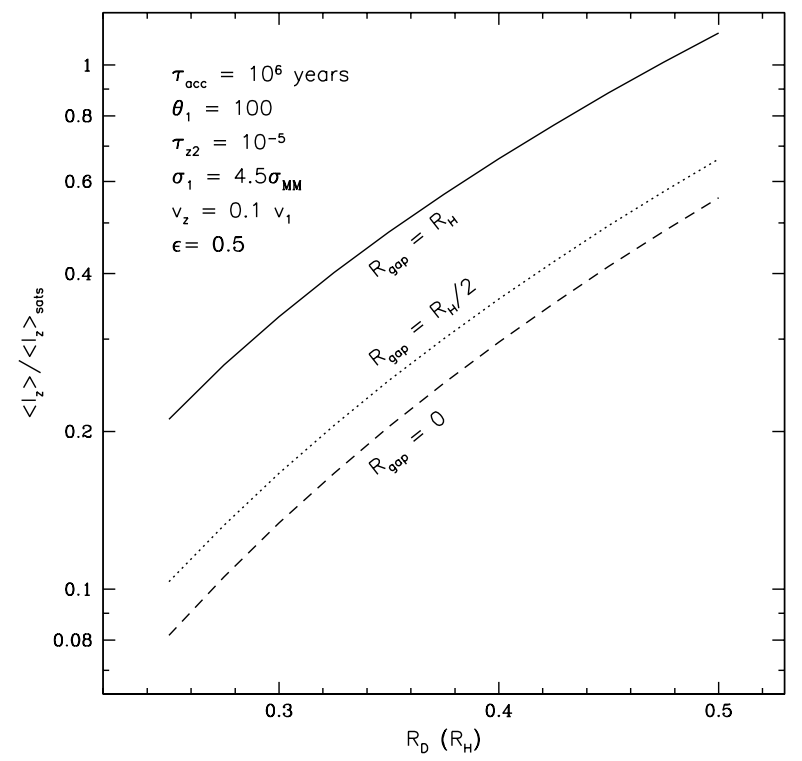

Figure 9: Specific angular momentum delivered in units of the specific angular momentum of the Galilean satellites $\left\langle\ell_{z}\right\rangle_{\text {sats }} \sim 1 \times 10^{17} \mathrm{~cm}^{2} \mathrm{~s}^{-1}$ as a function of circumplanetary disk size $R_{D}$ for three different values of the gap size $R_{\text {gap }}=\sqrt{\ell_{0} / \Omega_{1}}$, $R_{\text {gap }}=R_{H}$ (solid line), $R_{\text {gap }}=0.5 R_{H}$ (dotted line), and $R_{\text {gap }}=0$ (dashed line).

$$
\Lambda=\sigma_{1} \tau_{z 2}\left(\frac{v_{1}}{v_{z}}\right) \tau_{a c c}=\sigma_{1} P_{2}\left(\frac{v_{1}}{v_{z}}\right) N_{c}
$$

where $\sigma_{1}$ is taken to be in units of the solids MMSN $\left(\sigma_{\mathrm{MM}}=3.3 \mathrm{~g} \mathrm{~cm}^{-2}\right)$, and $\tau_{a c c}$ and $P_{2}$ are in years. The pairs of curves in Figure 7 correspond to $\Lambda=280(\epsilon=0.8$, $\left.\sigma_{1}=2.8 \sigma_{\mathrm{MM}}\right), \Lambda=450\left(\epsilon=0.5, \sigma_{1}=4.5 \sigma_{\mathrm{MM}}\right)$, and $\Lambda=1200\left(\epsilon=0.2, \sigma_{1}=12 \sigma_{\mathrm{MM}}\right)$. If we change the values of parameters we can obtain approximately the same curves as before by keeping $\Lambda$ constant. One can also consider longer feeding timescales, but such solutions might be unrealistic.

In Figure 8 we plot the specific angular momentum as a function of the gap size for parameters such that the mass and angular momentum of the Galilean satellites are obtained for $\left\langle\ell_{z}\right\rangle /\left\langle\ell_{z}\right\rangle_{\text {sats }}=1$. As one might expect, the specific angular momentum curves first increase with gap size, reach a maximum, and begin to decrease as the gap size chokes off the mass inflow. It is interesing to note that for the case of nearly perfect angular momentum deposition efficiency $\epsilon \sim 1$ it would seem possible to match the constraints even without a gap. However, it remains the case that to satisfy the system constraints more generally most of the angular momentum must be delivered as the giant planet opens a gap in the planetesimal distribution.

In Figure 9 we plot the specific angular momentum delivered as a function of the satellitesimal disk size $R_{D}$ for several values of the gap size. This figure shows that 
one may need an extended disk $\sim R_{H} / 2$ in order to be able to deliver sufficient mass and angular momentum to form the regular satellites of Jupiter (and Saturn ${ }^{16}$ ).

Finally, in Figures 10 and 11 we increase the size of the inner disk boundary $R_{0}$ and compare the relative behavior of the angular momentum and mass curves as we did before. Strictly speaking, this procedure would be tantamount to opening a gap in the heliocentric planetesimals in the limit of small velocity dispersion and weak gravity, which is inconsistent with the assumptions employed to obtain these curves. Nevertheless, because opening a gap would preferentially decrease the inflow rate close to the planet, it is still instructive to consider this case. Here we simply re-scale the size of the gap by $R_{g a p}=5 R_{0}$. The results are qualitatively similar to before, except that the effect on $\left\langle\ell_{z}\right\rangle$ is less pronounced and a solution could not be found in the case of $\epsilon=0.2$.

\section{Discussion and Conclusions}

We have focused on outstanding issues that a planetesimal collisional capture model faces before it can provide a viable alternative to the model developed in MEa,b. We have investigated whether the mass and angular momentum budget in the Galilean satellites and Titan may be accounted for by a model that delivers material to the circumplanetary disk in the same timescale as the feeding zone clearing timescale $\left(\lesssim 10^{5}\right.$ years, see, e.g., Charnoz and Morbidelli 2003). It is possible that inelastic collisions between planetesimals within the Hill radius of the giant planet form an extended $\left(\sim R_{H} / 2\right)$ circumplanetary satellitesimal swarm, which then sustains itself by collisionally capturing sufficiently small $(<1 \mathrm{~km})$ interlopers produced by the fragmentation of planetesimals following giant planet formation. This process might result in a comparable surface density of solids in the subnebula as in the nebula $\left(\sigma_{2} \sim 1 \mathrm{~g} \mathrm{~cm}^{-2}\right)$, and enough mass to form the Galilean satellites over several collisional cycles $\left(N_{c} \sim 10\right)$.

This may work only if a significant fraction of the mass in the satellites originates from the outer regions of the planet's feeding zone, which means that the model may have difficulties delivering enough material to simultaneously account for the total masses of the regular satellites of Jupiter and Saturn. Yet, because collisions from Roche-lobe interlopers can pump-up the angular momentum of the circumplanetary disk whether or not the interaction results in significant mass capture, we show that a planetesimal collisional capture model may deliver sufficient angular momentum to account for the angular momentum budget of the regular satellites provided enough mass is also delivered (which may require that a significant fraction of the planetesimal mass be in small $<1 \mathrm{~km}$ fragments with low inclination orbits, as well as a solids enhanced solar nebula). Still, the feeding zone clearing timescale, which in this model controls the satellite formation timescale, may be too short to accomodate the result

\footnotetext{
${ }^{16}$ Saturn's satellite system might require one to adapt the present formalism to the case of a giant planet with significant obliquity.
} 


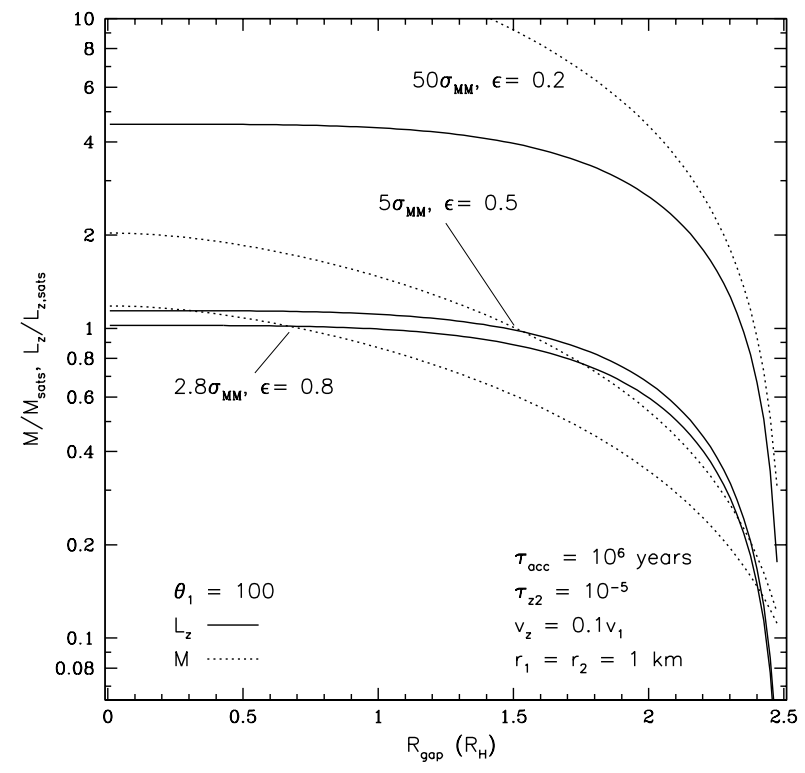

Figure 10: Mass (dotted lines) and angular momentum (solid lines) in units of the mass and angular momentum of the Galilean satellites $\left(M_{\text {sats }} \sim 4 \times 10^{26} \mathrm{~g}, L_{z, \text { sats }} \sim\right.$ $4 \times 10^{43} \mathrm{~g} \mathrm{~cm}^{2} \mathrm{~s}^{-1}$ ) accreted by the circumplanetary disk in $\tau_{a c c}=10^{6}$ years as a function of the gap size $R_{\text {gap }}=5 R_{0}$. For this case $N_{c}=10$, implying a steady-state disk mass is $M_{s w}=0.1 M_{s a t s}$. The proper budget of mass and angular momentum to account for the Galilean satellites is achieved where the solid and the dotted lines intersect at $L_{z} / L_{z, \text { sats }}=M / M_{\text {sats }}=1$.

that Callisto is partially differentiated (Anderson et al. 1998; 2001). This is because only if the satellite grows sufficiently slowly $\left(\tau_{a c c} \sim 10^{5}-10^{6}\right.$ years $)$ can it radiate away its accretion energy in time to keep it from melting and differentiating fully (Kuramoto and Matsui 1994). We have suggested that it may be possible to replenish the feeding zone of the giant planet with sufficiently small $(1-100 \mathrm{~m})$ planetesimal fragments brought in by gas drag, but we have not tested whether this is really possible. By this time the giant planet would presumably have opened a more or less clean gap in the gas disk, depending on the unknown turbulence parameter $\alpha$, which makes this a difficult problem to tackle. Also, resonances may capture inwardly drifting planetesimals, which might prevent them from replenishing the feeding zone. However, collisions or stirring by larger particles may free them and allow them to continue their inward migration. It is important to note that the model we advance here relies on fragmentation to decrease the size of planetesimals and make them easier to capture, but we do not employ collisional erosion directly as a capture mechanism.

Even if enough mass and angular momentum can be delivered, a planetesimal model still faces the further obstacle of how to constrain the model parameters. In particular, we have postponed modeling the planetesimal and satellitesimal mass spectrum, and have instead treated the size cutoffs for the planetesimals and satel- 


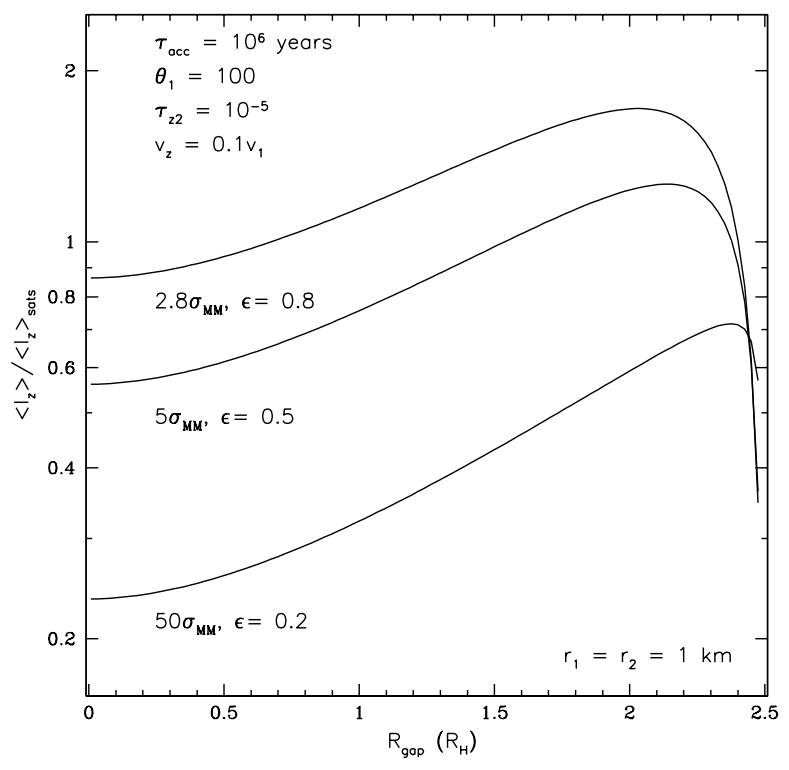

Figure 11: Specific angular momentum delivered in units of the specific angular momentum of the Galilean satellites $\left\langle\ell_{z}\right\rangle_{\text {sats }} \sim 1 \times 10^{17} \mathrm{~cm}^{2} \mathrm{~s}^{-1}$ as a function of gap size $R_{\text {gap }}=5 R_{0}$. These curves correspond to cases such that $\left\langle\ell_{z}\right\rangle /\left\langle\ell_{z}\right\rangle_{\text {sats }}=1$ matches the mass and angular momentum budget of the Galilean satellite system.

litesimals $\left(r_{1}\right.$ and $\left.r_{2}\right)$ as adjustable parameters, meant only to indicate characteristic sizes containing a significant fraction of the solids disk mass. Although we have found regions of parameter space that might plausibly lead to a self-consistent model for regular satellite formation, until reliable ways are found to constrain the relevant parameters, it remains uncertain how meaningful this is. In particular, it is difficult to know what to choose for the size of the circumplanetary disk particles $r_{2}$, where most of the satellitesimal mass resides (given our assumed value for $q_{2}=11 / 6$ ). One might argue that irregular satellites may provide an indication, but it is presently unknown whether the irregular satellites were collisionally captured, or bear any connection to the process that led to the formation of the regular satellites. On the other hand, we do not believe that observations of irregular satellite properties can be used to rule out the possibility either. Even if a connection can be made, however, subsequent collisional and dynamical evolution of satellitesimals would make it very difficult to extract useful constraints from the observed population of irregular satellites.

In theory, it might be possible to model the coupled problem of the fragmentation and growth of circumplanetary satellitesimals and heliocentric planetesimals, but at present we are far from realizing this goal. This raises the issue of what physical process sets the size for $r_{2}$. If this size is determined by a competition between collisional disruption by heliocentric interlopers and Safronov-type growth in the circumplanetary disk, then a difficulty arises for the model we advance here (at least for $r_{1} \gtrsim r_{2}$ ). Namely, our system constraints lead us to a model in which collisions 
between satellitesimals and similarly-sized planetesimals are more frequent than collisions between similarly-sized satellitesimals. This is because we seek a mass balance (i.e., satellitesimal collisions remove a similar amount of mass as gets delivered by captured planetesimals), yet hypervelocity collisions with similarly-sized interlopers do not always result in capture (i.e., $p(R)<<1$ ). As a result, satellitesimals may not have sufficient time to grow to the typical sizes $r_{2} \sim 1 \mathrm{~km}$ we use to satisfy our system constraints (which require long satellitesimal collisional times $\sim 10^{5}$ years, such that $\left.N_{c}=\tau_{\text {acc }} / \tau_{\text {coll }} \sim 10\right)$. However, it may be possible to circumvent this problem if we decrease the satellitesimal Safronov growth time by accreting "cold" circumplanetary debris, and increase $p(R)$ by capturing sufficiently small heliocentric planetesimals in low inclination orbits and large Safronov parameter $\theta_{1}>1$. It is also possible that the satellitesimal cutoff size is instead set by the sizes of the largest captured planetesimal fragments, though we have not yet investigated the implications of this possibility. It seems likely that a low velocity dispersion population of small planetesimal fragments, as might be expected at the time of satellite formation, might be needed in any case. Perhaps the most promising region of parameter space is one in which both the satellitesimal and planetesimal populations of interest can be best described as collisional rubble, so that $r_{2} \lesssim 0.1 \mathrm{~km}$, but we leave investigation of that regime for future work.

Much more work would need to be done to constrain this problem to the point where it can be stated whether a planetesimal collisional capture satellite formation model is viable. The arguments presented here constitute only the outline of a promising model. For instance, it is clearly an oversimplification to assume as we have done here that collisions between retrograde and prograde satellites control the evolution of the satellitesimal swarm in a timescale $\tau_{\text {coll }}$. Also, the capture probability $p(R)$ does not explicitly treat the physics of collisional fragmentation and may be unrealistic (we are currently considering other possible choices based on pi-scaling theory for the fractional mass captured following a disruptive collision). Furthermore, we have avoided the issue of the contribution to the angular momentum from regions of the disk (between $\sim 0.5-0.7 R_{H}$; e.g., Nesvorny et al. 2003) where only retrograde orbits are stable ${ }^{17}$ and prograde objects are rapidly lost. At this time, it is most useful to discuss how Cassini observations can affect this model's prospects compared to that of MEa,b (see also Appendix C). The main observational objection that might be levelled against this model is that it fails to account for the composition of Saturn's icy satellites. In particular, the medium-sized Saturnian satellites have densities that would seem to preclude a solar composition ice/rock ratio (this is only marginally true for Titan itself), which one might not expect from a model that derives the bulk of the solids that make-up the regular satellites directly from heliocentric orbit. This issue has been noted before in a number of publications (e.g., Johnson et al. 1987; Lunine and Tittemore 1993; Podolak et al. 1993) largely on the basis of a comparison

\footnotetext{
${ }^{17}$ One would need to argue that either the capture probability decreases outside $\sim R_{H} / 2$ or that most of this retrograde material is not delivered to the inner disk where regular satellites form but rather escapes outward.
} 
between the composition of regular satellites and objects thought to have formed in the outer solar system such as Pluto-Charon (see, e.g., McKinnon et al. 1997) as well as Triton (McKinnon and Mueller 1989). The recent Cassini flyby of Phoebe has yielded a density of $1.6 \mathrm{~g} \mathrm{~cm}^{-3}$ for this retrograde, irregular satellite (Porco et al. 2005), which indicates a rock to ice ratio of at least 50\% (Johnson and Lunine 2005), and reinforces the demarcation between objects that formed in the outer solar nebula and the giant planet subnebula.

Mosqueira and Estrada (2005) sketch a possible resolution of this issue, involving a hypervelocity collision between Titan and a Triton-sized differentiated interloper, and the accretion of secondary icy moons out of a volatile-rich disk formed in the aftermath of the collision. In this scenario Iapetus may have been scattered by Titan to its present position, and Hyperion may represent a remnant from the collision which became captured into resonance and was thus prevented from crossing Titan's orbit and getting either scattered or accreted by it (which was presumably the fate of other objects forming in the region). If so, one would expect an icy composition for both Iapetus and Hyperion. ${ }^{18}$ Regarding Titan itself, such an energetic impact would very likely have led to a fully differentiated state (Tonks and Melosh 1992), but there are a number of reasons to expect that Titan will turn out to be fully differentiated. Conversely, in the unlikely event that Cassini data indicated that Titan like Callisto is only partially differentiated that would argue against such an event, but in favor of a planetesimal collisional capture model, since it would provide evidence that the bulk of the material forming Titan was delivered over a long timescale as we assume here.

It is also important to consider the consequences of this model to the delivery of volatiles trapped in planetesimals to the atmosphere of Titan. This delivery of trapped volatiles is presumably also linked to the Galileo probe observation that Jupiter's heavy volatile element global abundances, excluding neon and oxygen, are about 3 times solar (Atreya et al. 1999; Mahaffy et al. 2000). Given that to form the clathrate of Ar, the model of Gautier et al. (2001a,b) requires a very low solar nebula temperature $T \sim 36 \mathrm{~K}$, which may be too cold for the location of Jupiter even for passive disk models, and the temperature constraint to trap it in amorphous ice is even lower $T \sim 25 K$ (Notesco and Bar-Nun 2005), the observed enhacement appears to argue that planetesimals trapped argon either as clathrate or in amorphous ice in cold regions of the disk before migrating to warmer regions. If so, our planetesimal collisional capture formation model would seem to imply $\mathrm{Ar} / \mathrm{N}_{2} \sim 0.06$ Owen (2000) and that the molecular nitrogen in Titan's atmosphere was delivered as trapped $N_{2}$. However, before such a conclusion can be reached one must first gain an understanding of the consequences of planetesimal collisional grinding on trapped volatiles.

\footnotetext{
${ }^{18}$ In the model of MEa,b one also expects an icy composition for Iapetus and Hyperion. In this model satellitesimals forming in the outer disk are icy (Mosqueira and Estrada 2005) and drift in a long timescale due to gas drag. As they approach a satellite they might get captured into resonances, and collisions among them might grind them down and knock them out of resonance. Thus, in this model too Hyperion might represent a collisional remnant. Also, one might expect that Hyperion-like objects might be typical impactors in the formation of Titan and Callisto.
} 


\section{Acknowledgements}

We thank Dr. Jeffrey N. Cuzzi for a thoughtful review of this manuscript and useful conversations during the course of this work. This work is supported by a grant from PGG and the National Research Council. 


\section{Appendix A: List of Symbols}

\begin{tabular}{ll}
\hline & Orbital period. \\
$P_{i}$ & Orbital frequency. \\
$\Omega_{i}$ & Probability of capture due to collisions with population $i$. \\
$p_{i}$ & Solids surface density. \\
$\sigma_{i}$ & Volume density of solids. \\
$\rho_{i}$ & Maximum cutoff in the size distribution. \\
$r_{i, m a x}$ & Minimum cutoff in the size distribution. \\
$r_{i, m i n}$ & Maximum cutoff in the size distribution. \\
$r_{i}$ & Maximum cutoff in the mass distribution. \\
$m_{i, m a x}$ & Minimum cutoff in the mass distribution. \\
$m_{i, m i n}$ & Mass distribution function. \\
$n_{i}$ & Proportionality constant for $n_{i}$. \\
$C_{i}$ & Power-law index for $n_{i}$. \\
$q_{i}$ & Scale-height of population $i$. \\
$H_{i}$ & Vertical optical depth when all particles are of size $r_{i, m a x}$. \\
$\tau_{z i}$ & Mean velocity of incoming planetesimals. \\
$v_{1}$ & Random velocity. \\
$v$ & Escape velocity from the giant planet. \\
$v_{e}$ & Safronov parameter. \\
$\theta_{1}$ & Planetesimal or satellitesimal density. \\
$\rho_{s}$ & Jacobi constant. \\
$C_{J}$ & Total collisional mass inflow rate. \\
$I$ & Contribution to $I$ due to satellitesimals smaller than incoming \\
$I_{s p}$ & planetesimals. \\
$I_{l b}$ & Contribution to $I$ due to satellitesimals larger than incoming \\
& planetesimals. \\
$r_{c}$ & Characteristic size for which all particles are captured into the \\
$R_{H}$ & circumplanetary disk. \\
$R_{P}$ & Hill radius of the giant planet. \\
$R_{0}$ & Radius of the giant planet. \\
$R_{D}$ & Inner boundary of the circumplanetary disk. \\
$N_{c}$ & Collisional cycles. \\
$n_{p}$ & Number of times a planetesimal crosses the circumplanetary disk \\
$\tau_{c o l l}$ & before being scattered. \\
$\tau_{a c c}$ & Collision time in the circumplanetary disk. \\
& Planetesimal delivery or accretion timescale. \\
&
\end{tabular}




\begin{tabular}{|c|c|}
\hline$\tau_{f r a g}$ & Fragmentation timescale for $r_{2, \max }$. \\
\hline$\tau_{e j}$ & Planetesimal ejection timescale. \\
\hline$G$ & Gravitational constant. \\
\hline$a$ & Distance to the Sun. \\
\hline$R$ & Distance to the giant planet. \\
\hline$M_{P}$ & Mass of the giant planet. \\
\hline$M_{\odot}$ & Mass of the Sun. \\
\hline$\mu$ & Ratio of secondary to primary. \\
\hline$\lambda_{G}$ & Mean free path of a gas molecule. \\
\hline$J_{i}$ & $\begin{array}{l}\text { Rate of objects in population } i \text { undergoing collisions per unit } \\
\text { volume. }\end{array}$ \\
\hline$\dot{\mathcal{F}}_{i}$ & Mass delivery rate per unit volume due to population $i$. \\
\hline$\dot{M}_{1}$ & $\begin{array}{l}\text { Mass delivery rate to the circumplanetary disk due to free-free } \\
\text { collisions. }\end{array}$ \\
\hline$\dot{\mathcal{F}}_{c s}$ & $\begin{array}{l}\text { Mass delivery rate per unit volume for collisions } \\
\text { between comparable-sized bodies. }\end{array}$ \\
\hline$\dot{M}$ & Mass accretion rate. \\
\hline$M_{s w}$ & Steady-state mass of the circumplanetary disk. \\
\hline$b$ & Ratio of disk location $R$ to Hill radius. \\
\hline$s$ & $\begin{array}{l}\text { Ratio of differential rotation in the circumplanetary disk } \\
\text { to the random velocities of incoming planetesimals. }\end{array}$ \\
\hline $\mathcal{I}$ & Total mass collisional rate per unit area \\
\hline $\mathcal{I}_{L}$ & Total angular momentum collisional rate per unit area \\
\hline$R_{\text {gap }}$ & Gap size in giant planet feeding zone. \\
\hline$L_{z}$ & z-component of the total angular momentum. \\
\hline$\ell_{z}$ & z-component of the specific angular momentum. \\
\hline$\ell_{0}$ & Specific angular momentum boundary value. \\
\hline$\left\langle\ell_{z}\right\rangle$ & Mean specific angular momentum. \\
\hline$\Phi\left(R, \ell_{z}\right)$ & Angular momentum distribution function. \\
\hline
\end{tabular}




\section{Appendix B: $4^{\text {th }}$ Order Expansion of $\Phi\left(R, \ell_{z}\right)$}

In this appendix, we derive the 4th order expansions for the mass and angular momentum collision rates per unit area which are described by the distribution $\Phi\left(R, \ell_{z}\right)$, where $\Phi\left(R, \ell_{z}\right) d \ell_{z}$ is the mass collision rate per unit area of planetesimals with $z$-component of specific angular momentum $\ell_{z}$ in the range $\ell_{z}$ and $\ell_{z}+d \ell_{z}$. Our treatment follows that of DT93 for the case of weak gravity and high velocity dispersion. The mass collision rate per unit area $\mathcal{I}(R)$ and the angular momentum collision rate per unit area $\mathcal{I}_{L}(R)$ correspond to the zeroth and first moments

$$
\mathcal{I}(R)=\frac{1}{2 \pi} \int \Phi\left(R, \ell_{z}\right) d \ell_{z}, \quad \mathcal{I}_{L}(R)=\frac{1}{2 \pi} \int \ell_{z} \Phi\left(R, \ell_{z}\right) d \ell_{z},
$$

where integration is over all angular momenta such that $\left|\ell_{z}\right| \geq \ell_{0}$.

In polar coordinates, the distribution funcion $\Phi\left(R, \ell_{z}\right)$ is given by Eq. (43) where the quantity $\Psi$ is

$$
\frac{1}{2} \Psi \equiv \frac{v^{2} \cos ^{2} \psi}{2 v_{1}^{2}}+\frac{\left[v \sin \psi-\Gamma \Omega_{1} R \cos (\chi+\psi)\right]^{2}}{2 v_{1}^{2}\left(1-\frac{1}{2} \Gamma\right)}=\frac{1}{2} \Psi_{0}-B_{1}+B_{2},
$$

where $\Psi_{0}=\left(v / v_{1}\right)^{2} g(\psi)$ and $g(\psi)$ is defined in Eq. (46), and $B_{1}$ and $B_{2}$ are given by

$$
B_{1}=\Gamma \frac{v}{v_{1}}\left(\frac{b}{s}\right) \frac{\sin \psi \cos (\chi+\psi)}{1-\frac{1}{2} \Gamma}, B_{2}=\frac{1}{2} \Gamma^{2}\left(\frac{b}{s}\right)^{2} \frac{\cos ^{2}(\chi+\psi)}{1-\frac{1}{2} \Gamma} .
$$

We derive an expression for the distribution function $\Phi\left(R, \ell_{z}\right) \propto e^{-\frac{1}{2} \Psi}=e^{-\frac{1}{2} \Psi_{0}} e^{B_{1}-B_{2}}$ by expanding the exponential in terms of $B_{1}$ and $B_{2}$ to fourth order in the quantity $b / s$. Using the trigonometric identity $\cos (\chi+\psi)=\cos \chi \cos \psi-\sin \chi \sin \phi$ and surpressing terms that are odd functions of sin and cos which will later vanish upon integration over $\psi$, the expansion is

$$
\begin{array}{r}
e^{B_{1}-B_{2}} \approx 1-2 f_{1} \frac{v}{v_{1}}\left(\frac{b}{s}\right) \sin \chi+\left(\frac{b}{s}\right)^{2}\left[2 f_{1}\left(\frac{v}{v_{1}}\right)^{2}-\Gamma\right]\left(f_{21}-2 f_{22} \sin ^{2} \chi\right) \\
-2\left(\frac{b}{s}\right)^{3}\left[2 f_{1}^{2}\left(\frac{v}{v_{1}}\right)^{3}-3 \Gamma f_{1}\left(\frac{v}{v_{1}}\right)\right]\left(f_{21}-f_{32} \sin ^{2} \chi\right) \sin \chi \\
+\left(\frac{b}{s}\right)^{4}\left[\frac{2}{3} f_{1}^{2}\left(\frac{v}{v_{1}}\right)^{4}-2 \Gamma f_{1}\left(\frac{v}{v_{1}}\right)^{2}+\frac{1}{2} \Gamma^{2}\right]\left(f_{41}-2 f_{42} \sin ^{2} \chi+f_{43} \sin ^{4} \chi\right),
\end{array}
$$

where the functions $f$ are given by

$$
\begin{aligned}
& f_{1}(g)=g(\psi)-1, \quad f_{21}(g)=\frac{1-\left(1-\frac{1}{2} \Gamma\right) g}{1-\frac{1}{2} \Gamma}, \quad f_{22}(g)=\frac{1-\Gamma / 4-\left(1-\frac{1}{2} \Gamma\right) g}{1-\frac{1}{2} \Gamma} \\
& f_{32}(g)=\frac{1+\frac{1}{3}\left(1-\frac{1}{2} \Gamma\right)-\frac{4}{3}\left(1-\frac{1}{2} \Gamma\right) g}{1-\frac{1}{2} \Gamma}, \quad f_{42}(g)=f_{41}(g)+\frac{3\left[1-\left(2-\frac{1}{2} \Gamma\right) g+\left(1-\frac{1}{2}\right) g^{2}\right]}{1-\frac{1}{2} \Gamma}, \\
& f_{41}(g)=\frac{\left[1-\left(1-\frac{1}{2} \Gamma\right) g\right]^{2}}{\left(1-\frac{1}{2} \Gamma\right)^{2}}, \quad f_{43}(g)=f_{41}(g)+\frac{6\left[1+\frac{1}{6}\left(1-\frac{1}{2} \Gamma\right)-\frac{1}{3}(7-2 \Gamma) g+\frac{7}{6}\left(1-\frac{1}{2} \Gamma\right) g^{2}\right]}{1-\frac{1}{2} \Gamma}
\end{aligned}
$$


Carrying out the integration over $\chi$ where $\sin \chi=\left(\ell_{z}-\Omega_{1} R^{2}\right) / v R$ leads to

$$
\begin{gathered}
\Phi\left(R, \ell_{z}\right)=\frac{2 \sigma_{1} \tau_{z 2}}{R\left[2 \pi\left(1-\frac{1}{2} \Gamma\right)\right]^{1 / 2} P_{1} v_{1}^{2} v_{z}} \int_{0}^{2 \pi} d \psi \int_{\left|\ell_{z}-\Omega_{1} R^{2}\right| / R}^{\infty} v e^{-\frac{1}{2} \Psi_{0}} \times \\
{\left[A_{0}+A_{2}\left(\frac{v}{v_{1}}\right)^{2}+A_{4}\left(\frac{v}{v_{1}}\right)^{4}+A_{-2}\left(\frac{v_{1}}{v}\right)^{2}+A_{-4}\left(\frac{v_{1}}{v}\right)^{4}\right] d v}
\end{gathered}
$$

where the $A_{i}$ are functions of the functions $f, \Gamma$, and $\alpha=\left(\ell_{z}-\Omega_{1} R^{2}\right) / \sqrt{2} v_{1} R$ arrived at by collecting of terms with common velocity powers in Eq. (B4). This expression can then be integrated over the velocity field to yield Eq. (45), where the coefficients $K_{i}$ are given by

$$
\begin{aligned}
& K_{0}=1-\left[\Gamma f_{21}-\frac{4}{g} f_{1} f_{21}\right]\left(\frac{b}{s}\right)^{2}+\left[\frac{1}{2} \Gamma^{2} f_{41}-\frac{4 \Gamma}{g} f_{1} f_{21}^{2}+\frac{16}{3 g^{2}} f_{1}^{2} f_{41}\right]\left(\frac{b}{s}\right)^{4}, \\
& K_{1}=-2 \sqrt{2} f_{1}\left(\frac{b}{s}\right)+\left[6 \sqrt{2} \Gamma f_{1} f_{21}-\frac{8 \sqrt{2}}{g} f_{1}^{2} f_{21}\right]\left(\frac{b}{s}\right)^{3}, \quad K_{2}=-4 f_{1}\left(2 f_{22}-f_{21}\right)\left(\frac{b}{s}\right)^{2} \\
& +4\left[\Gamma f_{1}\left(2 f_{42}-f_{41}\right)+\frac{g}{8} \Gamma^{2} f_{43}-\frac{4}{3 g} f_{1}^{2}\left(f_{42}-f_{41}\right)\right]\left(\frac{b}{s}\right)^{4}, \\
& K_{3}=8 \sqrt{2} f_{1}^{2}\left(f_{32}-f_{21}\right)\left(\frac{b}{s}\right)^{3}, \quad K_{4}=\frac{8}{3} f_{1}^{2}\left(f_{43}-2 f_{42}+f_{41}\right)\left(\frac{b}{s}\right)^{4}, \\
& K_{5}=2 g \Gamma f_{22}\left(\frac{b}{s}\right)^{2}-g \Gamma^{2} f_{42}\left(\frac{b}{s}\right)^{4}, \quad K_{6}=-6 g \sqrt{2} \Gamma f_{1} f_{32}\left(\frac{b}{s}\right)^{3}, \\
& K_{7}=-\left[4 g \Gamma f_{1} f_{43}+\frac{g^{2}}{2} \Gamma^{2} f_{43}\right]\left(\frac{b}{s}\right)^{4}
\end{aligned}
$$

The mass collision rate per unit area is then given by integration of $\Phi\left(R, \ell_{z}\right)$ over all $\left|\ell_{z}\right| \geq \ell_{0}$ and yields

$$
\mathcal{I}\left(R, \ell_{0}\right)=\frac{\sigma_{1} \tau_{z 2}}{\pi\left(1-\frac{1}{2} \Gamma\right)^{1 / 2} P_{1}} \frac{v_{1}}{v_{z}} \int_{0}^{2 \pi} \frac{d \psi}{[g(\psi)]^{3 / 2}} \sum_{i=0}^{7} \mathcal{M}_{i}\left(g(\psi), R, \ell_{0}\right),
$$

with the $\mathcal{M}_{i}$ given by

$$
\begin{aligned}
\mathcal{M}_{0} & =K_{0} h_{0}(\psi), \mathcal{M}_{1}=\frac{K_{1}}{2(\pi g)^{1 / 2}}\left(e^{-g \alpha_{0}^{2}}-e^{-g \alpha_{0}^{\prime 2}}\right)=K_{1} h_{1}(\psi) \\
\mathcal{M}_{2} & =K_{2}\left\{\frac{1}{2 g} h_{0}(\psi)+\frac{1}{2(\pi g)^{1 / 2}}\left(\alpha_{0} e^{-g \alpha_{0}^{2}}+\alpha_{0}^{\prime} e^{-g \alpha_{0}^{\prime 2}}\right)\right\}=K_{2}\left\{\frac{1}{2 g} h_{0}(\psi)+h_{2}(\psi)\right\} \\
\mathcal{M}_{3} & =\frac{K_{3}}{2(\pi g)^{1 / 2}}\left\{\left(\alpha_{0}^{2}+\frac{1}{g}\right) e^{-g \alpha_{0}^{2}}-\left(\alpha_{0}^{\prime 2}+\frac{1}{g}\right) e^{-g \alpha_{0}^{\prime 2}}\right\}=K_{3} h_{3}(\psi), \\
\mathcal{M}_{4} & =K_{4}\left\{\frac{3}{4 g^{2}} h_{0}(\psi)+\frac{1}{2(\pi g)^{1 / 2}}\left[\alpha_{0}\left(\alpha_{0}^{2}+\frac{3}{2 g}\right) e^{-g \alpha_{0}^{2}}+\alpha_{0}^{\prime}\left(\alpha_{0}^{\prime 2}+\frac{3}{2 g}\right) e^{-g \alpha_{0}^{\prime 2}}\right]\right\} \\
& =K_{4}\left\{\frac{3}{4 g^{2}} h_{0}(\psi)+h_{4}(\psi)\right\}, \quad \mathcal{M}_{5}=\frac{K_{5}}{3}\left\{\frac{1}{g} h_{0}(\psi)+2 h_{2}(\psi)+j_{3}(\psi)\right\} \\
\mathcal{M}_{6} & =\frac{K_{6}}{4}\left\{2 h_{3}(\psi)+j_{4}(\psi)\right\}, \quad \mathcal{M}_{7}=\frac{K_{7}}{5}\left\{\frac{3}{2 g^{2}} h_{0}(\psi)+2 h_{4}(\psi)+j_{5}(\psi)\right\}
\end{aligned}
$$


with $j_{n}(\psi)=(g / \pi)^{1 / 2}\left[\alpha_{0}^{n} \operatorname{Ei}\left(-g \alpha_{0}^{2}\right)+(-1)^{n+1} \alpha_{0}^{\prime n} \operatorname{Ei}\left(-g \alpha_{0}^{\prime 2}\right)\right]$, where the exponential integral $\operatorname{Ei}\left(-g \alpha^{2}\right)=-E_{1}\left(g \alpha^{2}\right)$, and

$$
h_{0}(\psi)=1-\frac{\operatorname{erf}\left(g^{1 / 2} \alpha_{0}\right)+\operatorname{erf}\left(g^{1 / 2} \alpha_{0}^{\prime}\right)}{2}
$$

Equation (B8) can now be integrated numerically over the angle $\psi$. Likewise, the $z$-component angular momentum collision rate per unit area is

$$
\begin{aligned}
& \mathcal{I}_{L}\left(R, \ell_{0}\right)=\frac{\sigma_{1} \tau_{z 2} \Omega_{1} R^{2}}{\pi\left(1-\frac{1}{2} \Gamma\right)^{1 / 2} P_{1}} \frac{v_{1}}{v_{z}} \times \\
& \left\{\int_{0}^{2 \pi} \frac{d \psi}{[g(\psi)]^{3 / 2}} \sum_{i=0}^{7} \mathcal{M}_{i}\left(g(\psi), R, \ell_{0}\right)+\frac{\sqrt{2} v_{1}}{\Omega_{1} R} \int_{0}^{2 \pi} \frac{d \psi}{[g(\psi)]^{3 / 2}} \sum_{i=0}^{7} \mathcal{L}_{i}\left(g(\psi), R, \ell_{0}\right)\right\}
\end{aligned}
$$

with the $\mathcal{L}_{i}$ given by

$$
\begin{gathered}
\mathcal{L}_{0}=\frac{K_{0}}{K_{1}} \mathcal{M}_{1}, \quad \mathcal{L}_{1}=\frac{K_{1}}{K_{2}} \mathcal{M}_{2}, \quad \mathcal{L}_{2}=\frac{K_{2}}{K_{3}} \mathcal{M}_{3}, \quad \mathcal{L}_{3}=\frac{K_{3}}{K_{4}} \mathcal{M}_{4}, \\
\mathcal{L}_{4}=K_{4} h_{5}(\psi), \mathcal{L}_{5}=\frac{K_{5}}{K_{6}} \mathcal{M}_{6}, \mathcal{L}_{6}=\frac{K_{6}}{K_{7}} \mathcal{M}_{7}, \\
\mathcal{L}_{7}=\frac{K_{7}}{6}\left\{4 h_{5}(\psi)+j_{6}(\psi)\right\} \\
h_{5}(\psi)=\frac{1}{2(\pi g)^{1 / 2}}\left\{\left[\left(\alpha_{0}^{2}+\frac{1}{g}\right)^{2}+\frac{1}{g^{2}}\right] e^{-g \alpha_{0}^{2}}-\left[\left(\alpha_{0}^{\prime 2}+\frac{1}{g}\right)^{2}+\frac{1}{g^{2}}\right] e^{-g \alpha_{0}^{\prime 2}}\right\} .
\end{gathered}
$$

Note that in the limit of $b / s<<1$, and $\alpha_{0}=\alpha_{0}^{\prime}=0$, the integrals over $\psi$ in Eq. (B8) and (B10) are identical to those in Eq. (44) and the expression for $\lambda=\left\langle\ell_{z}\right\rangle / \Omega R_{P}^{2}$ in Eq. (45) of DT93 (omitting the multiple-hits correction) with $R=R_{P}$ for the case of high dispersion and weak gravity. 


\section{Appendix C: Comparison Between the SEMM and GPPC Models}

\begin{tabular}{|c|c|c|}
\hline & SEMM Model & GPPC Model \\
\hline Callisto & $\begin{array}{l}\text { Consistent with partial } \\
\text { differentiation }\end{array}$ & $\begin{array}{l}\text { Consistent with partial } \\
\text { differentiation }\end{array}$ \\
\hline Ganymede & $\begin{array}{l}\text { Consistent with full } \\
\text { differentiation }\end{array}$ & $\begin{array}{l}\text { Consistent with full } \\
\text { differentiation }\end{array}$ \\
\hline Titan & Fully differentiated & $\begin{array}{l}\text { Partially differentiated (unless } \\
\text { a large collision took place) }\end{array}$ \\
\hline Iapetus & $\begin{array}{c}\text { Formed in-situ, consistent } \\
\text { with low density }\end{array}$ & $\begin{array}{l}\text { Inconsistent with low density } \\
\text { Formed closer in? }\end{array}$ \\
\hline Hyperion & $\begin{array}{l}\text { Icy satellitesimal } \\
\text { fragment }\end{array}$ & $\begin{array}{l}\text { Icy remnant of } \\
\text { a giant collision }\end{array}$ \\
\hline $\begin{array}{l}\text { Low density of } \\
\text { inner Saturnian } \\
\text { satellites }\end{array}$ & $\begin{array}{c}\text { Preferential loss of } \\
\text { silicates }\end{array}$ & $\begin{array}{l}\text { Possible Titan collision with } \\
\text { differentiated interloper }\end{array}$ \\
\hline $\begin{array}{c}\text { Compositional } \\
\text { gradient of Galilean } \\
\text { satellites }\end{array}$ & $\begin{array}{l}\text { Due to temperature } \\
\text { gradient of subnebula }\end{array}$ & $\begin{array}{l}\text { Laplace resonance } \\
\text { and/or stochastic } \\
\text { collisional component }\end{array}$ \\
\hline Titan's eccentricity & $\begin{array}{l}\text { Possibly satellite disk } \\
\text { interactions }\end{array}$ & $\begin{array}{l}\text { Possible collision } \\
\text { with Triton-sized } \\
\text { interloper }\end{array}$ \\
\hline Titan's isolation & $\begin{array}{l}\text { Survival by } \\
\text { gap-opening }\end{array}$ & $\begin{array}{l}\text { Break-up deep in } \\
\text { planetary potential } \\
\text { well }\end{array}$ \\
\hline $\begin{array}{l}\text { Titan's hydrocarbon } \\
\text { rich atmosphere }\end{array}$ & $\begin{array}{l}\text { Volatile rich } \\
\text { satellitesimals }\end{array}$ & $\begin{array}{l}\text { Volatiles present } \\
\text { in planetesimals }\end{array}$ \\
\hline $\begin{array}{l}\text { Size of disk from } \\
\text { which satellites } \\
\text { formed }\end{array}$ & $\begin{array}{l}\text { Specific angular } \\
\text { momentum of gas }\end{array}$ & $\begin{array}{c}\text { Specific angular } \\
\text { momentum of planetesimals }\end{array}$ \\
\hline
\end{tabular}




\begin{tabular}{|c|c|c|}
\hline $\begin{array}{l}\text { Satellite's } \\
\text { size-distance } \\
\text { correlation }\end{array}$ & Gas drag sorting & $\begin{array}{l}\text { Radial dependence } \\
\text { of isolation mass }\end{array}$ \\
\hline
\end{tabular}
Separation of Region is cleared An abundance of $\sim 1 \mathrm{~km}$ regulars and by gas drag objects yet to be irregulars found

\section{References}

Alfven, H., 1971. Motion of small particles in the solar system. In Physical Studies of Minor Planets, proceedings of IAU, colloq. 12 (ed. T. Gehrels), NASA SP 267, p. 315.

Alibert, Y., Mousis, O., 2004. Thermodynamical conditions and migration of protosatellites in the Jovian subnebula. 36th DPS meeting, 40.11.

Anderson, J. D., Schubert, G., Jacobson, R. A., Lau, E. L., Moore, W. B., Sjogren, W. L, 1998. Distribution of rock, metals, and ices in Callisto. Science 280, 1573 .

Anderson, J. D., Jacobson, R. A., McElrath, T. P., Moore, W. B., Schubert, G., Thomas, P. C., 2001. Shape, mean radius, gravity field, and interior structure of Callisto. Icarus 153, 157-161.

Atreya, S. K., Wong, M. H., Owen, T. C., Mahaffy, P. R., Niemann, H. B., De Pater, I., Drossart, P., Encrenaz, T., 1999. A comparison of the atmospheres of Jupiter and Saturn: deep atmospheric composition, cloud structure, vertical mixing, and origin. Plan. Space Sci. 47, 1243-1262.

Balbus, S. A., Hawley, J. F. 1998. Instability, turbulence, and enhanced transport in accretion disks. Rev. Modern Phys. 70, 1-53.

Bate, M. R., Lubow, S. H., Ogilvie, G. I., Miller, K. A., 2003. Three-dimensional calculations of high- and low-mass planets embedded in protoplanetary discs. Mon. Not. Roy. Astron. Soc. 341, 213-229.

Bryden, G., Chen, X., Lin, D. N. C., Nelson, R. P., Papaloizou, C. B. 1999. Tidally induced gap formation in protostellar disks: gap clearing and supression of protoplanetary growth. Astrophys. J. 514, 344-367.

Bryden, G., Rozyczka, M., Lin, D. N. C., Bodenheimer, P., 2000. On the interaction between protoplanets and protostellar disks. Astrophys. J. 540, 1091-1101.

CANUP, R. M., Ward, W. R., 2002. Formation of the Galilean satellites: conditions of accretion. Astron. J. 124, 3404-3423.

Charnoz, S., Morbidelli, A., 2003. Coupling dynamical and collisional evolution of small bodies: an application to the early ejection of planetesimals from the Jupiter-Saturn region. Icarus 166, 141-156. 
De, B. R., Alfven, H., Arrhenius, G., 1977. The critical velocity phenomenon and the origin of the regular satellites. In Planetary satellites. Tucson, University of Arizona Press, 1977, p. 490.

Dones, L., Tremaine, S., 1993. On the origin of planetary spins. Icarus 103, 67-92.

Dubrulle, B., 1993. Differential rotation as a source of angular momentum transport in the solar nebula. Icarus 106, 59-76.

Gautier, D., Hersant, F., Mousis, O., Lunine, J. I., 2001a. Enrichments in volatiles in Jupiter: A new interpretation of the Galileo measurements. Astrophys. J. 550, L227-L230.

Gautier, D., Hersant, F., Mousis, O., Lunine, J. I., 2001B. Erratum: Enrichments in volatiles in Jupiter: A new interpretation of the Galileo measurements. Astrophys. J. 559, L183-L183.

Hartmann, L., Calvet, N., Gullbring, E., D’Alessio, P. 1998. Accretion and the evolution of T Tauri disks. Astrophys. J. 495, 385.

Hawley, J. F., Balbus, S. A., Winters, W. F., 1999. Local hydrodynamic stability of accretion disks. Astrophys. J. 518, 394-404.

Hayashi, C. 1981. Structure of the solar nebula, growth and decay of magnetic fields, and effects of magnetic and turbulent viscosites on the nebula. Prog. Theor. Phys. Suppl. 70, 35-53.

Jacobson, R. A., Antreasian, P. G., Bordi, J. J., Criddle, K. E., Ionasescu, R., Jones, J. B., Mackenzie, R. A., Meek, M. C., Pelletier, F. J., Roth, D. C., Roundhill, I. M., Stauch, J. R., 2005. The orbits of the major Saturnian satellites and the gravity field of the Saturnian system. 36th DDA meeting, Santa Barbara, CA.

Johnson, T. V., Lunine, J. I., 2005. Density-derived constraints on the origin of Saturn's moon Phoebe. Nature, in press.

Johnson, T. V., Brown, R. H., Pollack, J. B., 1987. Uranus satellites - densities and composition. J. Geophys. Res. 92, 14884-14894.

Kenyon, S. J., Bromley, B. C., 2004. The size distribution of Kuiper belt objects. Astron. J. 128, 1916-1926.

Klahr, H. H., Bodenheimer, P., 2003. Turbulence in accretion disks: Vorticity generation and angular momentum transport via the global baroclinic instability. Astrophys. J. 582, 869.

Kuramoto, K., Matsui, T., 1994. Formation of a hot proto-atmosphere on the accreting giant icy satellite: Implications for the origin and evolution of Titan, Ganymede, and Callisto. J. Geophys. Res. 99, 21,183-21,200.

Li, H., J. M. Finn, R. V. E. Lovelace, Colgate, S. A., 2000. Rossby wave instability of thin accretion disks. II. Detailed linear theory. Astrophys. J. 533, 1023-1034.

Lissauer, J. J., Kary, D. M., 1991. The origin of the systematic component of planetary rotation. I - Planet on a circular orbit. Icarus 94, 126-159. 
Lubow, S. H., Seibert, M., Artymowicz, P., 1999. Disk accretion onto high-mass planets. Astrophys. J. 526, 1001.

Lunine. J. I., Stevenson, D. J., 1982. Formation of the Galilean satellites in a gaseous nebula. Icarus 52, 14-39.

Lunine, J. I., Tittemore, W. C., 1993. Origins of outer-planet satellites. In Protostars and Planets III, University of Arizona Press, Tucson, p. 1149-1176.

Mahaffy, P. R., Niemann, H. B., Alpert, A., Atreya, S. K., Demick, J., Donahue, T. M., Harpold, D. N., Owen, T. C., 2000. Noble gas abundance and isotope ratios in the atmosphere of Jupiter from the Galileo Probe Mass Spectrometer. J. Geophys. Res. 105, 15061-15072.

Makalkin, A. B., Dorofeeva, V. A., and Ruskol, E. L. 1999. Modeling the Protosatellite Circum-Jovian Accretion Disk: An Estimate of the Basic Parameters. Sol. Syst. Res. 33, p. 456.

McKinnon, W. B., Mueller, S., 1989. The density of Triton - A prediction. Geophys. Res. Lett 16, 591-594.

McKinnon, W. B., Simoneldi, D. P., Schubert, G., 1997. Composition, internal structure, and thermal evolution of Pluto and Charon. In Pluto and Charon, University of Arizona Press, Tucson, p. 295.

Mosqueira, I., Estrada, P. R., Chambers, J. E., 2000. Satellitesimal feeding and the formation of the regular satellites. American Astronomical Society, 32nd DPS meeting.

Mosqueira, I., Estrada, P. R., Cuzzi, J. N., Squyres, S. W., 2001. Circumjovian disk clearing after gap-opening and the formation of a partially differentiated Callisto. 32nd LPSC meeting, March 12-16, 2001, Houston, Texas, no. 1989.

Mosqueira, I., Kassinos, S., Shariff, K., Cuzzi, J. N., 2003. Hydrodynamical shear instability in accretion disks? American Astronomical Society, 35th DPS meeting $35,25.05$.

Mosqueira, I., Estrada, P. R. 2003A. Formation of the regular satellites of giant planets in an extended gaseous nebula I: subnebula model and accretion of satellites. Icarus 163, 198-231.

Mosqueira, I., Estrada, P. R. 2003B. Formation of the regular satellites of giant planets in an extended gaseous nebula II: satellite migration and survival. Icarus 163, 232-255.

Mosqueira, I., Estrada, P. R., 2005. On the origin of the Saturnian satellite system: Did Iapetus form in-situ? 36th LPSC meeting, Houston, TX, no. 1951.

Nesvorny, D., Alvarellos, J. L. A., Dones, L., Levison, H. F., 2003. Orbital and collisional evolution of the irregular satellites. Astron. J. 126, 398-429.

Notesco, G., BAr-Nun, A, 2005. A $25 \mathrm{~K}$ temperature of formation for the submicron ice grains which formed comets. Science, in press. 
OHtsuki, K., IdA, S., 1998. Planetary rotation by accretion of planetesimals with nonuniform spatial distribution formed by the planet's gravitational perturbation. Icarus 131, 393-420.

Owen, T. S., 2000. On the origin of Titan's atmosphere. Plan. Space Sci. 48, 747-752.

PAN, M., SARI, R., 2004. A generalization of the Lagrangian points: Studies of resonance for highly eccentric orbits. Astron. J. 128, 1418-1429.

Podolak, M., Hubbard, W. B., Pollack, J. B., 1993. Gaseous accretion and the formation of giant planets. In Protostars and Planets III, University of Arizona Press, Tucson, p. 1109-1147..

Pollack, J. B., Grossman, A. S., Moore, R., Graboske Jr., H. C., 1976. The formation of Saturn's satellites and rings as influenced by Saturn's contraction history. Icarus 29, 35-48.

Pollack, J. B., Lunine, J. I., Tittemore, W. C., 1991. Origin of the Uranian satellites. In Uranus. Tucson, University of Arizona Press, 1991, p. 469-512..

Porco, C. C., Baker, E., Barbara, J., Beurle, K., Brahic, A., Burns, J. A., Charnoz, S., Cooper, N., Dawson, D. D., Del Genio, A. D., Denk, T., Dones, L., Dyudina, U., Evans, M. W., Giese, B., Grazier, K., Helfenstein, P., Ingersoll, A. P., Jacobson, R. A., Johnson, T. V., McEwen, A., Murray, C. D., Neukum, G., Owen, W. M., Perry, J., Roatsch, T., Spitale, J., Squyres, S., Thomas, P. C., Tiscareno, M., Turtle, E., Vasavada, A. R., Veverka, J., Wagner, R., West, R., 2005. Cassini imaging science: Initial results on Phoebe and Iapetus. Science 307, 1237-1242.

RAfikov, R. R., 2002. Planet migration and gap formation by tidally induced shocks. Astrophys. J. 572, 566.

Richard, D., Zahn, J. P., 1999. Turbulence in differentially rotating flows. What can be learned from the Couette-Taylor experiment. Astron. Astrophys. 347, 734-738.

Rudiger, G., Arlt, R., Shalybkov, D., 2002. Hydrodynamic stability in accretion disks under the combined influence of shear and density stratification. Astron. Astrophys. 391, 781-787.

Ruskol, E. L., 1975. Origin of the Moon. NASA Transl. into english of the book "Proiskhozhdeniye Luny" Moscow, Nauka Press, 1975 p. 1-188.

Ruskol, E. L., 1981. Formation of planets. In ESA The Solar System and its Exploration, p. 107-113 (SEE N82-26087 16-88).

Ruskol, E. L., 1982. Origin of planetary satellites. Izves. Earth Phys. 18, 425-433.

Safronov, V. S., Ruskol, E. L., 1977. The accumulation of satellites. In Planetary Satellites. Tucson, University of Arizona Press, 1977, p. 501-512.

Safronov, V. S., Pechernikova, G. V., Ruskol, E. L., Vitiazev, A. V., 1986. Protosatellite Swarms. In Satellites Tucson, AZ, University of Arizona Press, 1986, p. $89-116$. 
Schmidt, O. Yu, 1957. Four lectures on the theory of the origin of the Earth. 3rd edition, Moscow, NA SSSR, press, 1957.

Schultz-Grunow, F., 1959. On the stability of Couette flow. Z. Angew. Math. Mech. 39, 101.

Shakura, N. I., Sunyaev, R. A., 1973. Black holes in binary systems. Observational appearance. Astron. Astrophys. 24, 337-355.

Shoemaker, E. M. 1984. Kuiper Prize Lecture, 16th DPS Meeting, Kona, HI.

Stern, S. A., Weissman, P. R., 2001. Rapid collisional evolution of comets during the formation of the Oort cloud. Nature 409, 589-591.

Stevenson, D. J., A. W. Harris, Lunine, J. I., 1986. Origins of satellites. In Satellites (J. A. Burns and M. S. Matthews, Eds.) Univ. of Arizona Press, Tucson.

TANAKA, H., IDA, S., 1997. Distribution of planetesimals around a protoplanet in the nebula gas II. Numerical simulations. Icarus 125, 302-316.

Tonks, W. B., Melosh, H. J., 1992. Core formation by giant impacts. Icarus 100, 326-346.

Weidenschilling, S. J., 1977. Aerodynamics of solid bodies in the solar nebula. Mon. Not. Roy. Astron. Soc. 180, 57-70.

Weidenschilling, S. J., 1997. The origin of comets in the solar nebula: A unified model. Icarus 127, 290-306.

Zahn, J. P., 1991. On the nature of disk viscosity. Proceedings of the IAU Colloq. 129, Structure and Emission Properties of Accretion Disks (C. Bertout, S. Collin-Souffrin, and J. P. Lasota, Eds.), Gif-sur-Yvette: Editions Frontieres, 1991, p.87.. 